\title{
Trans-Hydroalumination/Alkylation: One-Pot Synthesis of Trisubstituted Allylic Alcohols
}

\author{
Neil F. Langille and Timothy F. Jamison* \\ Department of Chemistry, Massachusetts Institute of Technology \\ Cambridge, MA 02139
}

\section{Supporting Information}

\section{General Methods:}

All reactions were performed in oven-dried glassware, under an oxygenfree argon atmosphere with rigid exclusion of moisture from reagents and solvents. Vitride (Red-Al ${ }^{\circledR}$ sodium bis(2-methoxyethoxy)aluminum hydride, $\geq 65$ wt. \% in toluene) and diisobutylaluminum hydride (reagent grade) were purchased from Aldrich Chemical Co., and used without further purification. lodomethane and iodoethane were purified by washing with aqueous sodium thiosulfate, water, and then aqueous sodium carbonate, followed by distillation and storage over copper powder. Methyllithium and $n$-butyllithium were titrated prior to use (menthol, $\mathrm{Et}_{2} \mathrm{O}, 0^{\circ} \mathrm{C}, 1,10$-phenanthroline indicator). 2,6-Lutidine was distilled over calcium hydride and stored over solid potassium hydroxide. Chlorotrimethylsilane and dichloromethane were distilled from calcium hydride. Tetrahydrofuran and ethyl ether were distilled from a purple solution of sodium benzophenone ketyl. All other reagents were used as supplied by the chemical manufacturers.

Analytical thin layer chromatography (TLC) was performed on silica gel 60 $\mathrm{F}_{254}$ aluminum plates precoated with a fluorescent indicator. Visualization of the developed chromatogram was accomplished with UV light $(254 \mathrm{~nm})$ and stained with either iodine-silica or vanillin. Data are reported as follows: $\mathrm{R}_{f}$ (solvent mixture, UV activity, color (stain)). Liquid chromatography was performed using a forced air-flow (flash chromatography ${ }^{\mathrm{S1}}$ ) on silica gel (Silicycle, 230-400 mesh), using the indicated solvent (underline denotes compound elution with indicated

(S1) Still, W. C.; Kahn, M.; Mitra, A. J. Org. Chem. 1978, 43, 2923-2925. 
solvent mixture $\left.(\mathrm{s})^{2}\right) . \quad{ }^{1} \mathrm{H}$ and ${ }^{13} \mathrm{C}$ NMR spectra were recorded, unless otherwise noted, in $\mathrm{CDCl}_{3}$ on a Varian Inova $500 \mathrm{MHz}$ spectrometer. Chemical shifts are reported in parts per million using the solvent internal standard (chloroform, 7.27 and $77.23 \mathrm{ppm}$, respectively). Data are reported as follows: chemical shift, integration, multiplicity $(\mathrm{s}=$ singlet, $\mathrm{d}=$ doublet, $\mathrm{t}=$ triplet, $\mathrm{q}=$ quartet, $\mathrm{m}=$ multiplet, app = apparent, $\mathrm{b}=$ broad), coupling constant (in $\mathrm{Hz}$ ). Infrared spectra were recorded on a Perkin-Elmer 2000 FT-IR. High resolution mass spectra were obtained on a Bruker Daltonics APEXII 3 Tesla Fourier Transform Mass Spectrometer by Dr. Li Li of the MIT Department of Chemistry Instrumentation Facility.

\section{Standard Procedures:}

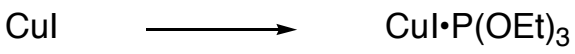

Sl1

Copper iodide triethylphosphite. Following Nishizawa's procedure,${ }^{\mathrm{S3}}$ copper (I) iodide $(5.00 \mathrm{~g}, 26.3 \mathrm{mmol})$ was added, slowly in portions over $10 \mathrm{~min}$, to a rt solution of triethylphosphite $(4.50 \mathrm{~mL}, 26.3 \mathrm{mmol}, 100 \mathrm{~mol} \%)$ in benzene $(26.3 \mathrm{~mL}, 1.00 \mathrm{M})$, maintaining the reaction temperature at approximately $25^{\circ} \mathrm{C}$. After the addition was complete, the reaction mixture was stirred, protected from light, for $1 \mathrm{~h}$. The crude mixture was filtered through Celite and concentrated in vacuo. The product was recrystallized from cyclohexane to afford $5.40 \mathrm{~g}(57.7 \%)$ desired product (SI1), displaying spectral properties in accord with literature precedent. The white crystalline material proved stable to storage at rt, protected from light, in a dessicator for several months. ${ }^{54}{ }^{1} \mathrm{H} \mathrm{NMR}\left(500 \mathrm{MHz}, \mathrm{CDCl}_{3}\right) \delta 4.12$ $(6 \mathrm{H}, \mathrm{q}, J=7.0), 1.30(9 \mathrm{H}, \mathrm{t}, J=7.0) .{ }^{\mathrm{S} 5}{ }^{13} \mathrm{C} \mathrm{NMR}\left(125 \mathrm{MHz}, \mathrm{CDCl}_{3}\right) \delta 61.1,16.8$.

(S2) Example: (silica; 2, $\underline{5}, \underline{10} \%$ EtOAc/hex) denotes silica as chromatography sorbent, $2 \%$ EtOAc/hex to elute impurities, desired compound elution at $5 \%$ and $10 \% \mathrm{EtOAc} / \mathrm{hex}$.

(S3) Nishizawa, Y. Bull. Chem. Soc. Jpn. 1961, 34, 1170-1178.

(S4) Decomposition of $\mathrm{Cul} \cdot \mathrm{P}(\mathrm{OEt})_{3}$ could be first noted by decreased solubility in THF.

(S5) Compare to P(OEt) $)_{3}:{ }^{1} \mathrm{H}$ NMR $\left(500 \mathrm{MHz}, \mathrm{CDCl}_{3}\right) \delta 3.88(6 \mathrm{H}, \mathrm{q}, J=7.0), 1.28(9 \mathrm{H}, \mathrm{t}, J=7.0)$. 


\section{General procedure for hydroalumination/alkylation with $\mathrm{Cul} \cdot \mathrm{P}(\mathrm{OEt})_{3}$ :}

A solution of propargyl alcohol $(1.0 \mathrm{mmol})$ in $\mathrm{Et}_{2} \mathrm{O}(2.0 \mathrm{~mL}, 0.50 \mathrm{M})$ was cooled to $0{ }^{\circ} \mathrm{C}$, and Vitride ( $\geq 65 \mathrm{wt}$. \% in toluene, $0.69 \mathrm{~mL}, 227 \mathrm{~mol} \%$ ) was added dropwise. The cold bath was removed and the reaction stirred for $6 \mathrm{~h}$. The flask was cooled to $0^{\circ} \mathrm{C}$ and $\mathrm{MeLi}\left(1.6 \mathrm{M}\right.$ in $\mathrm{Et}_{2} \mathrm{O}, 0.78 \mathrm{~mL}, 125 \mathrm{~mol} \%$ ) was added dropwise. The cold bath was removed and, after stirring for $90 \mathrm{~min}$, the reaction was cooled to $-78{ }^{\circ} \mathrm{C}\left(\mathrm{CO}_{2} /\right.$ acetone $)$. A solution of $\mathrm{Cul} \cdot \mathrm{P}(\mathrm{OEt})_{3}(357 \mathrm{mg}$, $100 \mathrm{~mol} \%)$ in THF (5.0 mL, $0.20 \mathrm{M}$ ) was added by cannula (exothermic!), followed by addition of neat electrophile $(300 \mathrm{~mol} \%)$. The reaction was stirred for $20 \mathrm{~h}$, allowing the cold bath to warm slowly to rt. The crude reaction mixture was poured onto sat. aq. potassium sodium tartrate $(50 \mathrm{~mL})$, rinsed with $40 \mathrm{~mL}$ EtOAc, and the mixture was stirred for $30 \mathrm{~min}$. The layers were separated, and the aqueous layer was extracted $2 x$ into $40 \mathrm{~mL}$ EtOAc. The combined organic layers were washed with brine, dried over $\mathrm{MgSO}_{4}$, filtered, and concentrated in vacuo. Purification by flash chromatography was generally required to afford pure allylic alcohol products

\section{General procedure for hydroalumination/alkylation with $\mathrm{CuCl}$ :}

A solution of propargyl alcohol $(1.0 \mathrm{mmol})$ in $\mathrm{Et}_{2} \mathrm{O}(2.0 \mathrm{~mL}, 0.50 \mathrm{M})$ was cooled to $0{ }^{\circ} \mathrm{C}$, and Vitride ( $\geq 65 \mathrm{wt}$. \% in toluene, $0.69 \mathrm{~mL}, 227 \mathrm{~mol} \%$ ) was added dropwise. The cold bath was removed and the reaction stirred for $6 \mathrm{~h}$. The flask was cooled to $0^{\circ} \mathrm{C}$ and $\mathrm{MeLi}\left(1.6 \mathrm{M}\right.$ in $\mathrm{Et}_{2} \mathrm{O}, 0.78 \mathrm{~mL}, 125 \mathrm{~mol} \%$ ) was added dropwise. The cold bath was removed and, after stirring for $90 \mathrm{~min}$, the reaction was cooled to $-78{ }^{\circ} \mathrm{C}\left(\mathrm{CO}_{2} /\right.$ acetone $)$. THF $(5.0 \mathrm{~mL}, 0.20 \mathrm{M})$ was added by syringe, followed by addition of neat electrophile $(300 \mathrm{~mol} \%)$ and solid $\mathrm{CuCl}$ (99 $\mathrm{mg}, 100 \mathrm{~mol} \%$ ). The reaction was stirred for $20 \mathrm{~h}$, allowing the cold bath to warm slowly to rt. The crude reaction mixture was poured onto sat. aq. potassium sodium tartrate $(50 \mathrm{~mL})$, rinsed with $40 \mathrm{~mL}$ EtOAc, and the mixture was stirred for $30 \mathrm{~min}$. The layers were separated, and the aqueous layer was extracted $2 \times$ into $40 \mathrm{~mL}$ EtOAc. The combined organic layers were washed with 
brine, dried over $\mathrm{MgSO}_{4}$, filtered, and concentrated in vacuo. Purification by flash chromatography was generally required to afford pure allylic alcohol products.

\section{Experimental Data and Procedures for New Compounds:}

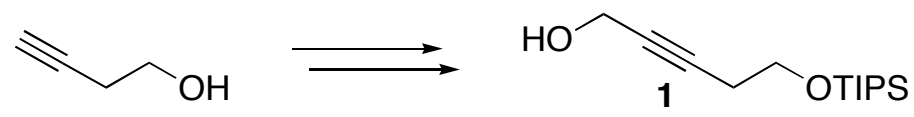

\section{5-(Triisopropylsilyloxy)pent-2-yn-1-ol (1).}

To a $0{ }^{\circ} \mathrm{C}$ solution of 3-butyn-1-ol $(2.00 \mathrm{~mL}, 26.4 \mathrm{mmol})$ in $\mathrm{CH}_{2} \mathrm{Cl}_{2}(66.0$ $\mathrm{mL}, 0.400 \mathrm{M})$ were added sequentially: 2,6-lutidine $(6.95 \mathrm{~mL}, 63.4 \mathrm{mmol}, 240$ $\mathrm{mol} \%)$ and TIPS-OTf $(8.55 \mathrm{~mL}, 31.7 \mathrm{mmol}, 120 \mathrm{~mol} \%)$. The reaction was stirred at $0{ }^{\circ} \mathrm{C}$ for $4 \mathrm{~h}$, then quenched by addition of $50 \mathrm{~mL}$ of water. The layers were separated, and the aqueous layer was extracted $3 \times$ into $50 \mathrm{~mL} \mathrm{CH}_{2} \mathrm{Cl}_{2}$. The combined organic layers were washed with brine, dried over $\mathrm{MgSO}_{4}$, filtered, and concentrated in vacuo. Purification by flash chromatography (silica; $\underline{0}, \underline{2} \%$ EtOAc/hex) provided the desired TIPS-protected alcohol as a colorless oil (5.80 g, 97.0\%). $R_{f} 0.90$ (5\% EtOAc/hex), no UV, brown ( $I_{2}$ stain).

Following Journet and Cai's procedure, ${ }^{\text {s6 }}$ the TIPS ether was dissolved in $64.0 \mathrm{~mL}$ THF $(0.400 \mathrm{M})$ and the solution was cooled to $-40^{\circ} \mathrm{C}$. $n$-Butyllithium (2.4 $\mathrm{M}$ in hexanes, $13.9 \mathrm{~mL}, 33.3 \mathrm{mmol}, 130 \mathrm{~mol} \%$ ) was added dropwise, and the mixture was stirred for $5 \mathrm{~min}$. $\mathrm{N}, \mathrm{N}$-Dimethylformamide $(4.36 \mathrm{~mL}, 56.3 \mathrm{mmol}$, $220 \mathrm{~mol} \%$ ) was added in one portion. After 1-2 min, the cold bath was removed and the reaction was allowed to stir $30 \mathrm{~min}$. Separately, the following were combined in a beaker, cooled to $0{ }^{\circ} \mathrm{C}$, and stirred vigorously: $11.6 \mathrm{~g} \mathrm{KHPO}_{4}, 104$ $\mathrm{mL} \mathrm{H}_{2} \mathrm{O}$, and $96.0 \mathrm{~mL}$ MTBE. After $30 \mathrm{~min}$, the reaction mixture was carefully poured onto the aq. $\mathrm{KHPO}_{4} / \mathrm{MTBE}$ mix, and stirred for $5 \mathrm{~min}$. The layers were separated, and the aqueous layers were extracted $3 \times$ into $100 \mathrm{~mL}$ MTBE. The combined organic layers were dried over $\mathrm{MgSO}_{4}$, filtered, concentrated in vacuo,

(S6) Journet, M.; Cai, D.; DiMichele, L. M.; Larsen, R. D. Tetrahedron Lett. 1998, 39, 6427-6428. 
and used crude in the next step. $\mathrm{R}_{f} 0.80$ (20\% EtOAc/hex), UV active, purple (vanillin).

The crude aldehyde was dissolved in $128 \mathrm{~mL} \mathrm{MeOH}(0.200 \mathrm{M})$ and cooled to $0{ }^{\circ} \mathrm{C}$. Solid $\mathrm{NaBH}_{4}(1.94 \mathrm{~g}, 200 \mathrm{~mol} \%)$ was added carefully, in portions over $5 \mathrm{~min}$. After $15 \mathrm{~min}$ at $0^{\circ} \mathrm{C}$, the reaction was carefully quenched with $50 \%$ aq. $\mathrm{NH}_{4} \mathrm{Cl}$ to a pH of 5 . The organic layer was extracted $3 \times$ into EtOAc. The combined organic layers were washed with brine, dried over $\mathrm{MgSO}_{4}$, filtered and concentrated. Purification by flash chromatography (silica; 5, $\underline{10}, \underline{20} \%$ EtOAc/hex) provided the title compound 1 as a colorless oil $(6.43 \mathrm{~g}, 97.9 \%$ yield over 2 steps). $R_{f} 0.20$ ( $20 \%$ EtOAc/hex), no UV, green (vanillin). ${ }^{1} \mathrm{H}$ NMR $\left(500 \mathrm{MHz}, \mathrm{CDCl}_{3}\right) \delta 4.25(2 \mathrm{H}, \mathrm{t}, J=2.1), 3.81(2 \mathrm{H}, \mathrm{t}, J=7.3), 2.48(2 \mathrm{H}, \mathrm{tt}$, $J=2.1,7.3), 1.51(1 \mathrm{H}, \mathrm{bs}), 1.06-1.07(21 \mathrm{H}, \mathrm{m}) .{ }^{13} \mathrm{C} \mathrm{NMR}\left(125 \mathrm{MHz}, \mathrm{CDCl}_{3}\right)$ $\delta 83.5,79.7,62.3,51.5,23.4,18.1,12.1$. IR (thin film NaCl) 3442, 2968, 2240, 1210, $1050 \mathrm{~cm}^{-1}$. HRMS ESI $(\mathrm{m} / \mathrm{z}):[\mathrm{M}+\mathrm{Na}]^{+}$calcd for $\mathrm{C}_{14} \mathrm{H}_{28} \mathrm{O}_{2} \mathrm{SiNa}, 279.1756$; found, 279.1750 .

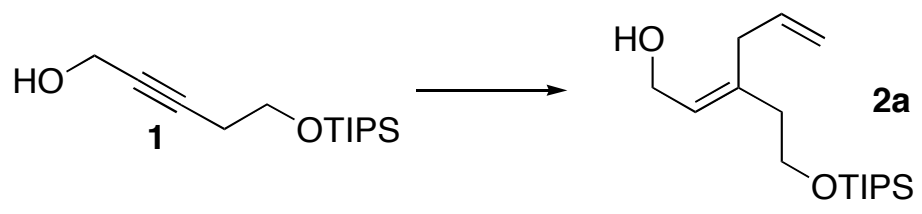

(E)-3-(2-(Triisopropylsilyloxy)ethyl)hexa-2,5-dien-1-ol (2a). Reaction was conducted with alkynol 1 (256 mg, $1.00 \mathrm{mmol})$ and allyl bromide $(0.26 \mathrm{~mL}$, $3.00 \mathrm{mmol}, 300 \mathrm{~mol} \%$ ), using standard $\mathrm{Cul} \cdot \mathrm{P}(\mathrm{OEt})_{3}$ or $\mathrm{CuCl}$ procedure. Purification by flash chromatography (silica; $5,10 \%$ EtOAc/hex) provided the title compound ( $86 \%$ and $97 \%$ yield, respectively). $R_{f} 0.80$ (30\% EtOAc/hex), faint UV, blue (vanillin). ${ }^{1} \mathrm{H} \mathrm{NMR}\left(500 \mathrm{MHz}, \mathrm{CDCl}_{3}\right) \delta 5.77$ (1H, ddt, $J=6.2,10.0$, 17.1), $5.55(1 \mathrm{H}, \mathrm{t}, J=6.9), 5.06(1 \mathrm{H}$, ddd, $J=1.8,3.5,17.1), 5.03(1 \mathrm{H}$, ddd, $J=1.5,3.3,10.0), 4.17(2 \mathrm{H}, \mathrm{d}, J=6.9), 3.77(2 \mathrm{H}, \mathrm{t}, J=7.1), 2.87(2 \mathrm{H}, \mathrm{d}$, $J=6.2), 2.30(2 \mathrm{H}, \mathrm{t}, J=7.1), 1.36(1 \mathrm{H}, \mathrm{bs}), 1.05-1.06(21 \mathrm{H}, \mathrm{m}) .{ }^{13} \mathrm{C} \mathrm{NMR}$ $\left(125 \mathrm{MHz}, \mathrm{CDCl}_{3}\right) \delta 138.5,136.1,126.7,115.8,62.7,59.2,40.5,35.5,18.2,12.1$. 
IR (thin film $\mathrm{NaCl}$ ) 3468, 3081, 2928, 1642, 1218, $1030 \mathrm{~cm}^{-1}$. HRMS ESI $(\mathrm{m} / \mathrm{z})$ : $[\mathrm{M}+\mathrm{Na}]^{+}$calcd for $\mathrm{C}_{17} \mathrm{H}_{34} \mathrm{O}_{2} \mathrm{SiNa}, 321.2226$; found, 321.2222 .

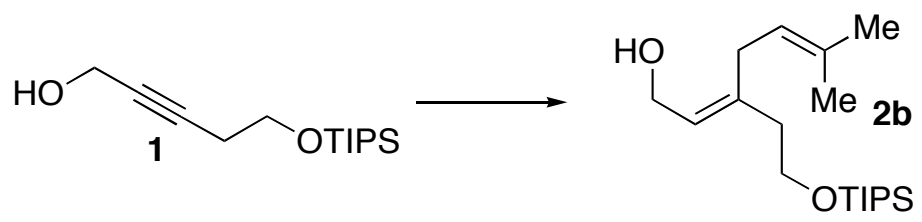

(E)-6-Methyl-3-(2-(triisopropylsilyloxy)ethyl)hepta-2,5-dien-1-ol

(2b).

Reaction was conducted with alkynol 1 (128 $\mathrm{mg}, 0.500 \mathrm{mmol}$ ) and 3,3dimethylallyl bromide $(0.17 \mathrm{~mL}, 1.50 \mathrm{mmol}, 300 \mathrm{~mol} \%)$, using standard Cul. $\mathrm{P}(\mathrm{OEt})_{3}$ or $\mathrm{CuCl}$ procedure. Purification by flash chromatography (silica; 2, 5\% EtOAc/hex) provided the title compound ( $81 \%$ and $82 \%$ yield, respectively). $\mathrm{R}_{f} 0.30$ (20\% EtOAc/hex), faint UV, blue (vanillin). ${ }^{1} \mathrm{H} \mathrm{NMR}\left(500 \mathrm{MHz}, \mathrm{CDCl}_{3}\right)$ $\delta 5.46(1 \mathrm{H}, \mathrm{t}, J=6.9), 5.02(1 \mathrm{H}, \mathrm{t}, J=7.1), 4.17(2 \mathrm{H}, \mathrm{d}, J=6.9), 3.76(2 \mathrm{H}, \mathrm{t}$, $J=7.2), 2.79(2 \mathrm{H}, \mathrm{d}, J=7.1), 2.27(2 \mathrm{H}, \mathrm{t}, J=7.2), 1.69(3 \mathrm{H}, \mathrm{s}), 1.66(3 \mathrm{H}, \mathrm{s}), 1.18$ $(1 \mathrm{H}, \mathrm{bs}), 1.06(21 \mathrm{H}, \mathrm{m}) .{ }^{13} \mathrm{C} \mathrm{NMR}\left(125 \mathrm{MHz}, \mathrm{CDCl}_{3}\right) \delta$ 143.4, 138.1, 127.3, 111.6, 62.7, 59.3, 40.4, 39.3, 22.6, 18.2, 12.1. IR (thin film $\mathrm{NaCl}$ ) 3460, 3060, 2941, 1380, 1365, 1212, $1030 \mathrm{~cm}^{-1}$. HRMS ESI $(\mathrm{m} / \mathrm{z}):[\mathrm{M}+\mathrm{Na}]^{+}$calcd for $\mathrm{C}_{19} \mathrm{H}_{38} \mathrm{O}_{2} \mathrm{SiNa}$, 349.2539; found, 349.2537.

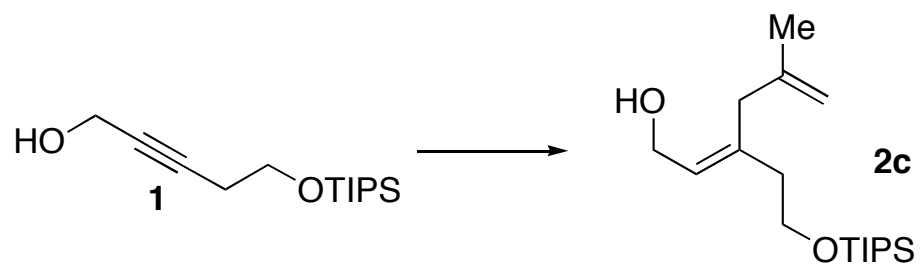

(E)-5-Methyl-3-(2-(triisopropylsilyloxy)ethyl)hexa-2,5-dien-1-ol (2c). Reaction was conducted with alkynol 1 (128 mg, $0.500 \mathrm{mmol}$ ) and 3-bromo-2methylpropene $(0.15 \mathrm{~mL}, 1.50 \mathrm{mmol}, 300 \mathrm{~mol} \%)$, using standard $\mathrm{Cul} \cdot \mathrm{P}(\mathrm{OEt})_{3}$ or $\mathrm{CuCl}$ procedure. Purification by flash chromatography (silica; $\underline{4}, \underline{6} \% \mathrm{EtOAc/hex}$ ) provided the title compound ( $84 \%$ and $94 \%$ yield, respectively). $R_{f} 0.30(20 \%$ EtOAc/hex), faint UV, blue (vanillin). ${ }^{1} \mathrm{H} N M R\left(500 \mathrm{MHz}, \mathrm{CDCl}_{3}\right) \delta 5.62(1 \mathrm{H}, \mathrm{t}$, $J=6.5), 4.79(1 \mathrm{H}, \mathrm{d}, J=0.7), 4.72(1 \mathrm{H}, \mathrm{d}, J=0.7), 4.15(2 \mathrm{H}, \mathrm{t}, J=6.5), 3.77(2 \mathrm{H}$, 
$\mathrm{t}, J=7.0), 2.81(2 \mathrm{H}, \mathrm{s}), 2.27(2 \mathrm{H}, \mathrm{t}, J=7.0), 1.70(3 \mathrm{H}, \mathrm{s}), 1.05-1.06(21 \mathrm{H}, \mathrm{m})$. ${ }^{13} \mathrm{C}$ NMR $\left(125 \mathrm{MHz}, \mathrm{CDCl}_{3}\right) \delta 143.4,138.1,127.3,111.6,62.7,59.3,40.4,39.3$, 22.6, 18.2, 12.1. IR (thin film NaCl) 3462, 3078, 2929, 1220, $1035 \mathrm{~cm}^{-1}$. HRMS ESI ( $m / z):[\mathrm{M}+\mathrm{Na}]^{+}$calcd for $\mathrm{C}_{18} \mathrm{H}_{36} \mathrm{O}_{2} \mathrm{SiNa}, 335.2382$; found, 335.2386.

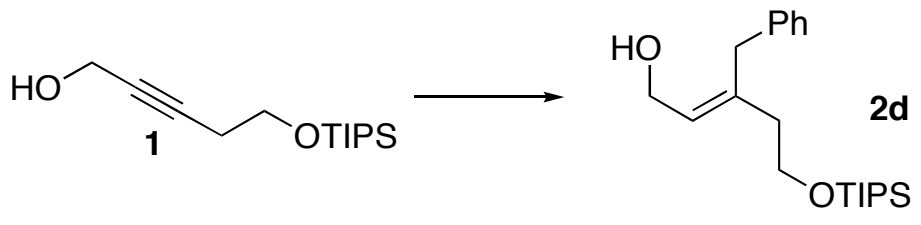

(E)-3-Benzyl-5-(triisopropylsilyloxy)pent-2-en-1-ol (2d). Reaction was conducted with alkynol 1 (128 $\mathrm{mg}, 0.500 \mathrm{mmol})$ and benzyl bromide $(0.18 \mathrm{~mL}$, $1.50 \mathrm{mmol}, 300 \mathrm{~mol} \%)$, using standard $\mathrm{Cul} \cdot \mathrm{P}(\mathrm{OEt})_{3}$ or $\mathrm{CuCl}$ procedure. Purification by flash chromatography (silica; 4 , $\underline{6} \%$ EtOAc/hex) provided the title compound (62\% and $60 \%$ yield, respectively). $R_{f} 0.30$ (20\% EtOAc/hex), faint UV, blue (vanillin). ' ${ }^{1} \mathrm{H} \mathrm{NMR}\left(500 \mathrm{MHz}, \mathrm{CDCl}_{3}\right) \delta$ 7.19-7.31 $(5 \mathrm{H}, \mathrm{m}), 5.66(1 \mathrm{H}, \mathrm{t}$, $J=7.0), 4.29(2 \mathrm{H}, \mathrm{m}), 3.72(2 \mathrm{H}, \mathrm{t}, J=6.9), 3.48(2 \mathrm{H}, \mathrm{s}), 2.23(2 \mathrm{H}, \mathrm{t}, J=6.9)$, 1.03-1.04 $(21 \mathrm{H}, \mathrm{m}) .{ }^{13} \mathrm{C} \mathrm{NMR}\left(125 \mathrm{MHz}, \mathrm{CDCl}_{3}\right) \delta 138.3,129.5,129.2,126.6$, 126.5, 62.5, 59.1, 40.2, 38.6, 18.4, 12.0. IR (thin film $\mathrm{NaCl}$ ) 3446, 3030, 2942,1210, 1035, $850 \mathrm{~cm}^{-1}$. HRMS ESI $(\mathrm{m} / \mathrm{z}):[\mathrm{M}+\mathrm{Na}]^{+}$calcd for $\mathrm{C}_{21} \mathrm{H}_{36} \mathrm{O}_{2} \mathrm{SiNa}$, 371.2382 ; found, 371.2381.

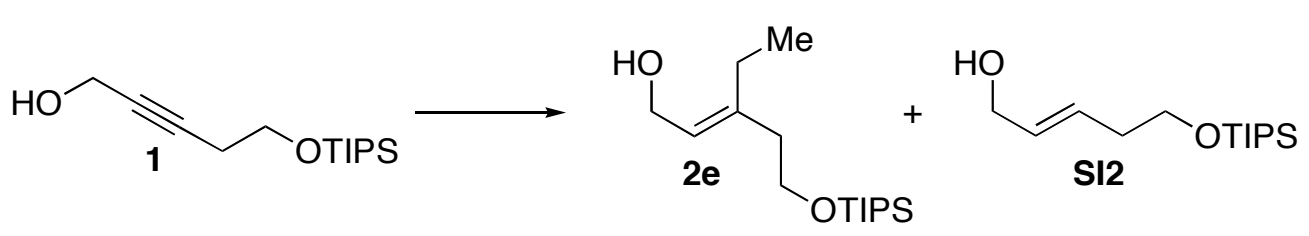

$\begin{array}{lll}\text { (E)-3-ethyl-5-(triisopropylsilyloxy)pent-2-en-1-ol (2e). Reaction was } & \text { (2e) }\end{array}$ conducted with alkynol 1 (128 $\mathrm{mg}, 0.500 \mathrm{mmol})$ and iodoethane $(0.12 \mathrm{~mL}$, $1.50 \mathrm{mmol}, 300 \mathrm{~mol} \%)$, using standard $\mathrm{Cul} \cdot \mathrm{P}(\mathrm{OEt})_{3}$ or $\mathrm{CuCl}$ procedure. ${ }^{1} \mathrm{H}$ NMR analysis of the crude reaction mixture revealed a mixture of desired product $\mathbf{2 e}$ and proton quench (SI2, see scheme above). Careful purification by flash chromatography (silica; 5, $8 \%$ EtOAc/hex) provided the title compound ( $31 \%$ and 
$14 \%$ yield from Cul· $P(O E t)_{3}$ and $\mathrm{CuCl}$, respectively). $\mathrm{R}_{f} 0.25$ (20\% EtOAc/hex), faint UV, blue/purple (vanillin). ${ }^{1} \mathrm{H}$ NMR $\left(500 \mathrm{MHz}, \mathrm{CDCl}_{3}\right) \delta 5.89(1 \mathrm{H}, \mathrm{t}, J=5.9)$, $4.18(2 \mathrm{H}, \mathrm{t}, J=5.9), 3.81(2 \mathrm{H}, \mathrm{m}), 2.76(2 \mathrm{H}, \mathrm{m}), 1.82(2 \mathrm{H}, \mathrm{q}, J=7.9), 1.05-1.12$ $(22 \mathrm{H}, \mathrm{m}), 0.94(3 \mathrm{H}, \mathrm{m}) .{ }^{13} \mathrm{C}$ NMR $\left(125 \mathrm{MHz}, \mathrm{CDCl}_{3}\right) \delta 137.8,125.8,62.3,59.4$, 40.0, 25.0, 18.3, 12.1, 11.9. IR (thin film $\mathrm{NaCl}$ ) 3442, 3061, 2932, 1215, $1052 \mathrm{~cm}^{-1}$. HRMS ESI $(\mathrm{m} / \mathrm{z})$ : $[\mathrm{M}+\mathrm{Na}]^{+}$calcd for $\mathrm{C}_{16} \mathrm{H}_{34} \mathrm{O}_{2} \mathrm{SiNa}, 309.2226$; found, 309.2223.

(E)-5-(triisopropylsilyloxy)pent-2-en-1-ol (SI-2). ${ }^{1} \mathrm{H} \mathrm{NMR}\left(500 \mathrm{MHz}, \mathrm{CDCl}_{3}\right)$ $\delta 5.73(2 \mathrm{H}, \mathrm{m}), 4.16(2 \mathrm{H}, \mathrm{d}, J=7.0), 3.73(2 \mathrm{H}, \mathrm{m}), 2.31(2 \mathrm{H}, \mathrm{m}), 1.07(21 \mathrm{H}, \mathrm{m})$.

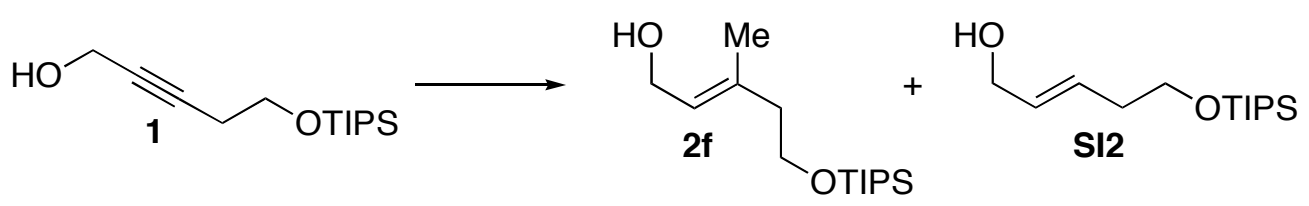

(E)-3-Methyl-5-(triisopropylsilyloxy)pent-2-en-1-ol (2f). Reaction was conducted with alkynol $1(128 \mathrm{mg}, 0.500 \mathrm{mmol})$ and iodomethane $(94 \mu \mathrm{L}$, $1.50 \mathrm{mmol}, 300 \mathrm{~mol} \%)$, using standard $\mathrm{Cul} \cdot \mathrm{P}(\mathrm{OEt})_{3}$ or $\mathrm{CuCl}$ procedure. ${ }^{1} \mathrm{H}$ NMR analysis of the crude reaction mixture revealed a mixture of desired product $\mathbf{2 f}$ and proton quench (SI2, see scheme above). Careful purification by flash chromatography (silica; 5, $8 \%$ EtOAc/hex) provided the title compound (44\% and $31 \%$ yield from Cul•P(OEt) ${ }_{3}$ and $\mathrm{CuCl}$, respectively). $\mathrm{R}_{f} 0.25$ (20\% EtOAc/hex), faint UV, blue (vanillin). ${ }^{1} \mathrm{H} \mathrm{NMR}\left(500 \mathrm{MHz}, \mathrm{CDCl}_{3}\right) \delta 5.93(1 \mathrm{H}, \mathrm{t}, J=5.9), 4.19$ $(2 \mathrm{H}, \mathrm{t}, J=5.9), 3.82(2 \mathrm{H}, \mathrm{t}, J=6.4), 2.74(2 \mathrm{H}, \mathrm{t}, J=6.4), 1.59(3 \mathrm{H}, \mathrm{s}), 1.05(21 \mathrm{H}$, m). $\left.{ }^{13} \mathrm{C} \mathrm{NMR} \mathrm{(125} \mathrm{MHz,} \mathrm{CDCl}_{3}\right) \delta 135.9,127.1,61.9,58.9,42.6,18.9,18.4,12.3$. IR (thin film NaCl) 3440, 3060, 2935, 1213, $1051 \mathrm{~cm}^{-1}$. HRMS ESI $(\mathrm{m} / \mathrm{z}):[\mathrm{M}+\mathrm{Na}]^{+}$ calcd for $\mathrm{C}_{15} \mathrm{H}_{32} \mathrm{O}_{2} \mathrm{SiNa}$, 295.2069; found, 295.2069.

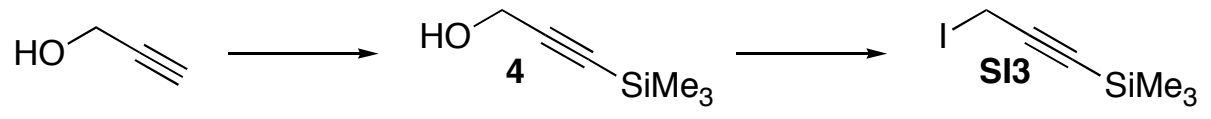

(3-lodoprop-1-ynyl)trimethylsilane (SI3). 
A solution of propargyl alcohol $(1.00 \mathrm{~mL}, 17.2 \mathrm{mmol})$ in THF $(43.0 \mathrm{~mL}$, $0.400 \mathrm{M}$ ) was cooled to $-78^{\circ} \mathrm{C}$, and $n$-BuLi $(2.4 \mathrm{M}$ in hexanes, $21.5 \mathrm{~mL}$, $51.5 \mathrm{mmol}, 300 \mathrm{~mol} \%)$ was added dropwise. After stirring for $20 \mathrm{~min}$ at $-78{ }^{\circ} \mathrm{C}$, TMS-Cl $(7.70 \mathrm{~mL}, 60.1 \mathrm{mmol}, 350 \mathrm{~mol} \%)$ was added dropwise. The reaction was stirred for $5 \mathrm{~min}$ at $-78{ }^{\circ} \mathrm{C}$, the cold bath was removed, and the reaction stirred $1 \mathrm{~h}$. The reaction was quenched with $50 \mathrm{~mL}$ water and then $10 \%$ aq. $\mathrm{HCl}$ was added to the crude reaction mixture until complete consumption of the TMS-ether was observed (TLC analysis, $\mathrm{R}_{f} 0.90$ (10\% EtOAc/hex), no UV, purple (vanillin)). The reaction was poured into a separatory funnel containing $100 \mathrm{~mL}$ $\mathrm{Et}_{2} \mathrm{O}$, shaken, and the layers were separated. The aqueous layer was extracted $2 \times$ into $100 \mathrm{~mL} \mathrm{Et} \mathrm{E}_{2} \mathrm{O}$. The combined organic layers were washed with brine, dried over $\mathrm{MgSO}_{4}$, filtered, and carefully concentrated in vacuo (10 torr, $0^{\circ} \mathrm{C}$ ). Purification by flash chromatography (silica; $5, \underline{20} \% \mathrm{Et}_{2} \mathrm{O} /$ pent) provided the alcohol (4) (2.15 g, 97.6\%) as a pale yellow oil. $\mathrm{R}_{f} 0.25$ (10\% EtOAc/hex), no UV, purple (vanillin). ${ }^{1} \mathrm{H}$ NMR (500 MHz, $\left.\mathrm{CDCl}_{3}\right) \delta 4.28(2 \mathrm{H}, \mathrm{s}), 1.62(1 \mathrm{H}, \mathrm{bs}), 0.19$ $(9 \mathrm{H}, \mathrm{s}) .{ }^{13} \mathrm{C}$ NMR $\left(125 \mathrm{MHz}, \mathrm{CDCl}_{3}\right) \delta 104.2,90.5,51.5,-0.1$. IR (thin film $\mathrm{NaCl}$ ) 3430, 2200, $1036 \mathrm{~cm}^{-1}$. HRMS ESI $(\mathrm{m} / \mathrm{z}):[\mathrm{M}+\mathrm{Na}]^{+}$calcd for $\mathrm{C}_{6} \mathrm{H}_{12} \mathrm{OSiNa}$, 151.0555; found, 151.0553.

A solution of $\mathrm{PPh}_{3}\left(313 \mathrm{mg}, 1.20 \mathrm{mmol}, 120 \mathrm{~mol} \%\right.$ ) in $2.50 \mathrm{~mL} \mathrm{CH} \mathrm{Cl}_{2}$ was cooled to $0{ }^{\circ} \mathrm{C}$. To the resulting solution were added sequentially: imidazole (102 mg, $1.50 \mathrm{mmol}, 150 \mathrm{~mol} \%$ ), iodine (342 mg, $1.35 \mathrm{mmol}, 135 \mathrm{~mol} \%$ ), and a solution of alcohol 4 (128 mg, $1.00 \mathrm{mmol})$ in $\mathrm{CH}_{2} \mathrm{Cl}_{2}(2.50 \mathrm{~mL}, 0.200 \mathrm{M}$ total). The reaction was stirred at $0{ }^{\circ} \mathrm{C}$, protected from light, for $90 \mathrm{~min}$. The reaction was quenched by addition of $35 \mathrm{~mL} 20 \%$ aq. $\mathrm{Na}_{2} \mathrm{~S}_{2} \mathrm{O}_{3}$ and $35 \mathrm{~mL} \mathrm{CH}_{2} \mathrm{Cl}_{2}$. The resulting layers were separated. The aqueous layer was extracted $2 \times$ into $35 \mathrm{~mL}$ $\mathrm{CH}_{2} \mathrm{Cl}_{2}$. The combined organic layers were washed with brine, dried over $\mathrm{MgSO}_{4}$, filtered, and carefully concentrated in vacuo $\left(10\right.$ torr, $\left.0^{\circ} \mathrm{C}\right)$. Purification by flash chromatography (silica; pent) provided the target iodide SI3 (150 mg, $63.2 \%)$ as a pale yellow oil. $R_{f} 0.90$ (5\% EtOAc/hex), UV, faint brown (vanillin). ${ }^{1} \mathrm{H}$ NMR $\left(500 \mathrm{MHz}, \mathrm{CDCl}_{3}\right) \delta 3.71(2 \mathrm{H}, \mathrm{s}), 0.17(9 \mathrm{H}, \mathrm{s})$. The propargyl iodide 
proved somewhat unstable, and was utilized immediately in subsequent reactions.

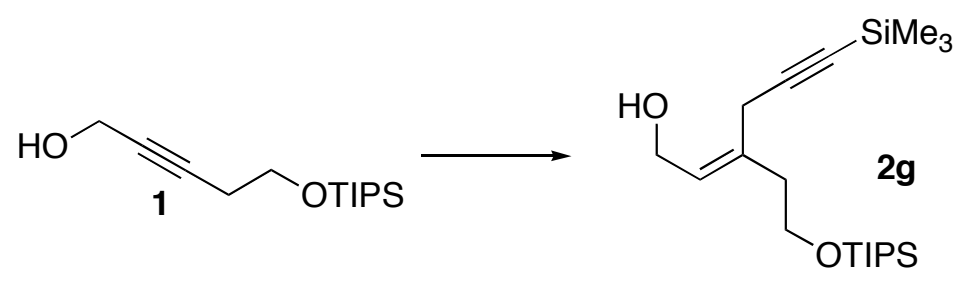

(E)-3-(2-(Triisopropylsilyloxy)ethyl)-6-(trimethylsilyl)hex-2-en-5-yn-1-ol (2g).

Reaction was conducted with alkynol 1 (128 mg, $0.500 \mathrm{mmol}$ ) and propargyl iodide SI3 (357 mg, $1.50 \mathrm{mmol}, 300 \mathrm{~mol} \%$ ), using standard $\mathrm{Cul} \cdot \mathrm{P}(\mathrm{OEt})_{3}$ or $\mathrm{CuCl}$ procedure. Purification by flash chromatography (silica; 4 , $\underline{6} \%$ EtOAc/hex) provided the title compound ( $37 \%$ and $22 \%$ yield, respectively). $R_{f} 0.30(20 \%$ EtOAc/hex), faint UV, blue (vanillin). ${ }^{1} \mathrm{H} N M R\left(500 \mathrm{MHz}, \mathrm{CDCl}_{3}\right) \delta 5.53(1 \mathrm{H}, \mathrm{t}$, $J=6.8), 4.18(2 \mathrm{H}, \mathrm{d}, J=6.8), 3.75(2 \mathrm{H}, \mathrm{t}, J=7.2), 2.72(2 \mathrm{H}, \mathrm{s}), 2.32(2 \mathrm{H}, \mathrm{t}$, $J=7.2), 1.41\left(1 \mathrm{H}\right.$, bs), 1.03-1.05 $(21 \mathrm{H}, \mathrm{m}), 0.20(9 \mathrm{H}, \mathrm{s}) .{ }^{13} \mathrm{C} \mathrm{NMR}(125 \mathrm{MHz}$, $\left.\mathrm{CDCl}_{3}\right) \delta 138.6,123.0,101.2,90.5,63.1,59.4,40.3,23.1,18.0,12.1,-0.1$. IR (thin film NaCl) $3436,3065,2930,2225,1220,1060 \mathrm{~cm}^{-1}$. HRMS ESI $(\mathrm{m} / \mathrm{z})$ : $[\mathrm{M}+\mathrm{Na}]^{+}$calcd for $\mathrm{C}_{20} \mathrm{H}_{40} \mathrm{O}_{2} \mathrm{SiNa}, 391.2465$; found, 391.2450.

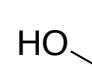

$\mathrm{HO}$

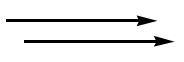

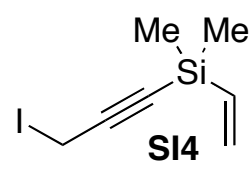

\section{(3-lodoprop-1-ynyl)dimethyl(vinyl)silane (SI4).}

A solution of propargyl alcohol $(0.50 \mathrm{~mL}, 8.6 \mathrm{mmol})$ in THF $(22 \mathrm{~mL}$, $0.40 \mathrm{M}$ ) was cooled to $-78{ }^{\circ} \mathrm{C}$ and $n$-BuLi $(2.4 \mathrm{M}$ in hexanes, $10.7 \mathrm{~mL}$, $25.8 \mathrm{mmol}, 300 \mathrm{~mol} \%)$ was added dropwise. After stirring for $20 \mathrm{~min}$ at $-78^{\circ} \mathrm{C}$, vinyldimethylchlorosilane $(4.10 \mathrm{~mL}, 30.1 \mathrm{mmol}, 350 \mathrm{~mol} \%)$ was added dropwise. The reaction was stirred for $30 \mathrm{~min}$ at $-78{ }^{\circ} \mathrm{C}$, the cold bath was removed, and the reaction stirred $5 \mathrm{~h}$. The reaction was quenched with $50 \mathrm{~mL}$ water and then $10 \%$ aq. $\mathrm{HCl}$ was added to the crude reaction mixture until complete consumption of the silyl ether was observed (TLC analysis, $R_{f} 0.90(10 \%$ EtOAc/hex), faint UV, blue (vanillin)). The reaction was poured into a separatory 
funnel containing $50 \mathrm{~mL} \mathrm{Et} \mathrm{t}_{2} \mathrm{O}$, shaken, and the layers were separated. The aqueous layer was extracted $2 \times$ into $50 \mathrm{~mL} \mathrm{Et}_{2} \mathrm{O}$. The combined organic layers were washed with brine, dried over $\mathrm{MgSO}_{4}$, filtered, and carefully concentrated in vacuo $\left(10\right.$ torr, $0^{\circ} \mathrm{C}$ ). Purification by flash chromatography (silica; $5, \underline{10}, \underline{20} \%$ $\mathrm{Et}_{2} \mathrm{O}$ /pent) provided the alkynyl silane $(763 \mathrm{mg}, 63.3 \%)$ as a pale yellow oil. $\mathrm{R}_{f} 0.25$ (10\% EtOAc/hex), faint UV, light purple (vanillin). ${ }^{1} \mathrm{H} \mathrm{NMR}(500 \mathrm{MHz}$, $\left.\mathrm{CDCl}_{3}\right) \delta 6.14(1 \mathrm{H}, \mathrm{dd}, J=14.0,19.5), 6.03(1 \mathrm{H}, \mathrm{dd}, J=4.0,14.0), 5.85(1 \mathrm{H}, \mathrm{dd}$, $J=4.0,19.5), 4.30(2 \mathrm{H}, \mathrm{s}), 1.62(1 \mathrm{H}, \mathrm{bs}), 0.26(6 \mathrm{H}, \mathrm{s}) .{ }^{13} \mathrm{C} \mathrm{NMR}(125 \mathrm{MHz}$, $\left.\mathrm{CDCl}_{3}\right) \delta 136.3,133.5,105.2,88.7,51.6,-1.6$.

A solution of $\mathrm{PPh}_{3}(224 \mathrm{mg}, 0.713 \mathrm{mmol}, 120 \mathrm{~mol} \%)$ in $1.78 \mathrm{~mL} \mathrm{CH} \mathrm{Cl}_{2}$ was cooled to $0{ }^{\circ} \mathrm{C}$. To the resulting solution were added sequentially: imidazole (72 mg, $1.07 \mathrm{mmol}, 150 \mathrm{~mol} \%$ ), iodine (244 mg, $0.963 \mathrm{mmol}, 135 \mathrm{~mol} \%$ ), and alkynyl silane $(100 \mathrm{mg}, 0.713 \mathrm{mmol})$ in $\mathrm{CH}_{2} \mathrm{Cl}_{2}(1.78 \mathrm{~mL}, 0.200 \mathrm{M}$ total). The reaction was stirred at $0{ }^{\circ} \mathrm{C}$, protected from light, for $45 \mathrm{~min}$. The reaction was quenched by addition of $35 \mathrm{~mL} 20 \%$ aq. $\mathrm{Na}_{2} \mathrm{~S}_{2} \mathrm{O}_{3}$ and $35 \mathrm{~mL} \mathrm{CH}_{2} \mathrm{Cl}_{2}$. The resulting layers were separated. The aqueous layer was extracted $2 \times$ into $35 \mathrm{~mL}$ $\mathrm{CH}_{2} \mathrm{Cl}_{2}$. The combined organic layers were washed with brine, dried over $\mathrm{MgSO}_{4}$, filtered, and carefully concentrated $\left(10\right.$ torr, $\left.0^{\circ} \mathrm{C}\right)$. Purification by flash chromatography (silica; pent) provided the target iodide SI4 (142 mg, 79.4\%) as a pale yellow oil. $\mathrm{R}_{f} 0.90$ ( $5 \%$ EtOAc/hex), UV, faint brown (vanillin). ${ }^{1} \mathrm{H}$ NMR $\left(500 \mathrm{MHz}, \mathrm{CDCl}_{3}\right) \delta 6.13(1 \mathrm{H}, \mathrm{dd}, J=14.5,20.0), 6.04(1 \mathrm{H}, \mathrm{dd}, J=4.0,14.5)$, $5.86(1 \mathrm{H}, \mathrm{dd}, J=4.0,20.0), 3.73(2 \mathrm{H}, \mathrm{s}), 0.24(6 \mathrm{H}, \mathrm{s})$. The propargyl iodide proved somewhat unstable, and was utilized immediately in subsequent reactions. 


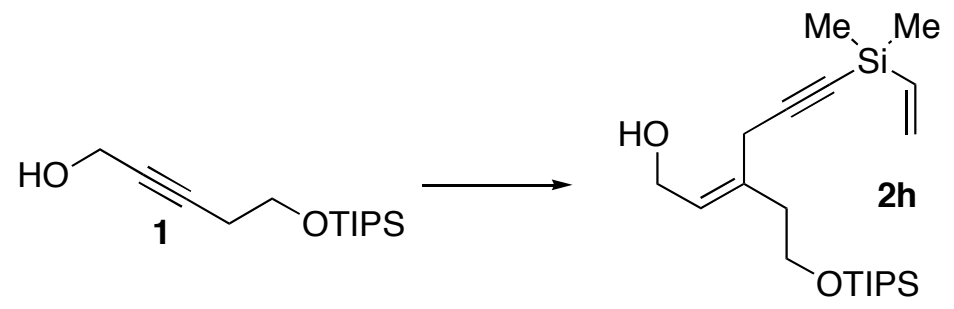

(E)-6-(Dimethyl(vinyl)silyl)-3-(2-(triisopropylsilyloxy)ethyl)hex-2-en-5-yn-1-ol

(2h). Reaction was conducted with alkynol 1 (128 mg, $0.500 \mathrm{mmol}$ ) and propargyl iodide S14 (375 mg, $1.50 \mathrm{mmol}, 300 \mathrm{~mol} \%$ ), using standard $\mathrm{Cul} \cdot \mathrm{P}(\mathrm{OEt})_{3}$ or $\mathrm{CuCl}$ procedure. Purification by flash chromatography (silica; $\underline{4}$, 6\% EtOAc/hex) provided the title compound (62\% and $47 \%$ yield, respectively). $\mathrm{R}_{f} 0.30$ (20\% EtOAc/hex), faint UV, purple (vanillin). ${ }^{1} \mathrm{H} \mathrm{NMR}\left(500 \mathrm{MHz}, \mathrm{CDCl}_{3}\right.$ ) $\delta 6.09(1 \mathrm{H}, \mathrm{dd}, J=13.8,19.5), 5.99(1 \mathrm{H}, \mathrm{dd}, J=4.0,13.8), 5.85(1 \mathrm{H}, \mathrm{dd}, J=4.0$, 19.5), $5.55(1 \mathrm{H}, \mathrm{t}, J=6.9), 4.20(2 \mathrm{H}, \mathrm{d}, J=6.9), 3.75(2 \mathrm{H}, \mathrm{t}, J=7.2), 2.71(2 \mathrm{H}$, s), $2.33(2 \mathrm{H}, \mathrm{t}, J=7.2), 1.04-1.06(21 \mathrm{H}, \mathrm{m}), 0.25(6 \mathrm{H}, \mathrm{s}) .{ }^{13} \mathrm{C} \mathrm{NMR}(125 \mathrm{MHz}$, $\left.\mathrm{CDCl}_{3}\right) \delta 138.5,133.3,123.1,105.1,88.5,62.9,59.4,40.1,23.3,17.9,12.1,-1.7$, -1.7. IR (thin film NaCl) 3446, 3060, 2927, 2228, 1660, 1220, $1062 \mathrm{~cm}^{-1}$. HRMS ESI $(m / z):[\mathrm{M}+\mathrm{Na}]^{+}$calcd for $\mathrm{C}_{21} \mathrm{H}_{40} \mathrm{O}_{2} \mathrm{Si}_{2} \mathrm{Na}, 403.2465$; found, 403.2463 .

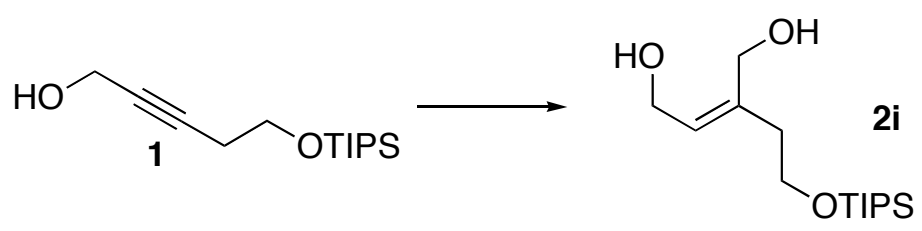

(Z)-2-(2-(Triisopropylsilyloxy)ethyl)but-2-ene-1,4-diol (2i). Reaction was conducted with alkynol 1 (128 $\mathrm{mg}, 0.500 \mathrm{mmol}$ ) and para-formaldehyde (45.0 mg, $1.50 \mathrm{mmol}, 300 \mathrm{~mol} \%$ ), using standard $\mathrm{Cul} \cdot \mathrm{P}(\mathrm{OEt})_{3}$ or $\mathrm{CuCl}$ procedure. Purification by flash chromatography (silica; 2, $\underline{5} \%$ EtOAc/hex) provided the title compound (55\% and $71 \%$ yield, respectively). $\mathrm{R}_{f} 0.10$ (30\% EtOAc/hex), no UV, purple (vanillin). ${ }^{1} \mathrm{H} \mathrm{NMR}\left(500 \mathrm{MHz}, \mathrm{CDCl}_{3}\right) \delta 5.63(1 \mathrm{H}, \mathrm{t}$, $J=6.9), 4.18(2 \mathrm{H}, \mathrm{d}, J=6.9), 4.15(2 \mathrm{H}, \mathrm{s}), 3.84(2 \mathrm{H}, \mathrm{t}, J=5.7), 2.39(2 \mathrm{H}, \mathrm{t}$, $J=5.7), 1.06-1.07(21 \mathrm{H}, \mathrm{m}) .{ }^{13} \mathrm{C}$ NMR $\left(125 \mathrm{MHz}, \mathrm{CDCl}_{3}\right) \delta 141.6,128.8,64.6$, 
60.6, 58.6, 39.7, 18.1, 12.0. IR (thin film NaCl) 3520, 3060, 1220, $1060 \mathrm{~cm}^{-1}$. HRMS ESI ( $\mathrm{m} / \mathrm{z})$ : $[\mathrm{M}+\mathrm{Na}]^{+}$calcd for $\mathrm{C}_{15} \mathrm{H}_{32} \mathrm{O}_{3} \mathrm{SiNa}, 311.2018$; found, 311.2016.

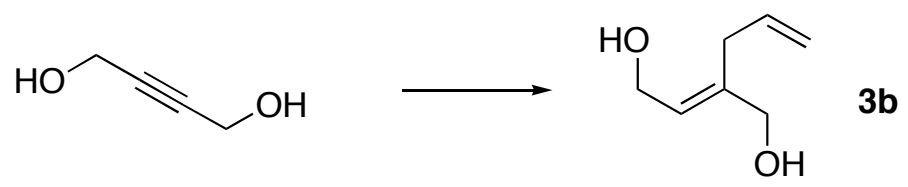

(E)-2-Allylbut-2-ene-1,4-diol (3b). Reaction was conducted with 2-butyn-1,4-diol (86.1 mg, $1.00 \mathrm{mmol})$ and allyl bromide (0.26 mL, $3.00 \mathrm{mmol}, 300 \mathrm{~mol} \%)$, using standard $\mathrm{Cul} \cdot \mathrm{P}(\mathrm{OEt})_{3}$ or $\mathrm{CuCl}$ procedure. Purification by flash chromatography (silica; 30, 100\% EtOAc/hex) provided the title compound (82\% and $89 \%$ yield, respectively). $\quad \mathrm{R}_{f} 0.30$ (50\% EtOAc/hex), no UV, purple (vanillin). ${ }^{1} \mathrm{H} N M R$ $\left(500 \mathrm{MHz}, \mathrm{CDCl}_{3}\right) \delta 5.75-5.78(2 \mathrm{H}, \mathrm{m}), 5.06(1 \mathrm{H}, \mathrm{dd}, J=1.5,18.0), 5.03(1 \mathrm{H}, \mathrm{dd}$, $J=1.5,10.5), 4.22(2 \mathrm{H}, \mathrm{d}, J=7.0), 4.06(2 \mathrm{H}, \mathrm{s}), 2.87(2 \mathrm{H}, \mathrm{d}, J=6.4), 2.17(2 \mathrm{H}$, m). ${ }^{13} \mathrm{C}$ NMR $\left(125 \mathrm{MHz}, \mathrm{CDCl}_{3}\right) \delta 140.0,135.6,125.4,116.1,66.2,59.0,32.7$. IR (thin film NaCl) 3506, 3082, 2927, $1630 \mathrm{~cm}^{-1}$. HRMS ESI $(\mathrm{m} / \mathrm{z})$ : $[\mathrm{M}+\mathrm{Na}]^{+}$calcd for $\mathrm{C}_{7} \mathrm{H}_{12} \mathrm{O}_{2} \mathrm{Na}, 151.0735$; found, 151.0739 .

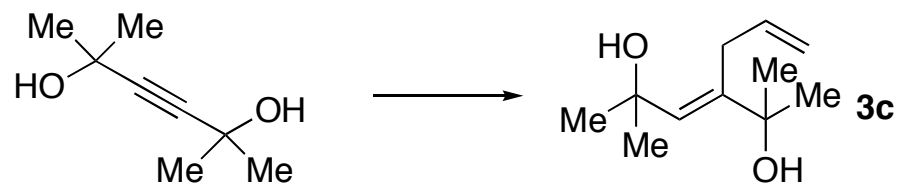

(E)-3-Allyl-2,5-dimethylhex-3-ene-2,5-diol (3c). Reaction was conducted with 2,5-dimethyl-3-hexyne-2,5-diol (39 mg, $0.273 \mathrm{mmol}$ ) and allyl bromide (71 $\mu \mathrm{L}$, $0.819 \mathrm{mmol}, 300 \mathrm{~mol} \%)$, using standard $\mathrm{Cul} \cdot \mathrm{P}(\mathrm{OEt})_{3}$ or $\mathrm{CuCl}$ procedure. Purification by flash chromatography (silica; 20, 30\% EtOAc/hex) provided the title compound ( $82 \%$ and $91 \%$ yield, respectively). $R_{f} 0.50$ ( $80 \%$ EtOAc/hex), no UV, blue (vanillin). ${ }^{1} \mathrm{H}$ NMR $\left(500 \mathrm{MHz}, \mathrm{CDCl}_{3}\right) \delta 6.05(1 \mathrm{H}, \mathrm{m}), 5.75(1 \mathrm{H}, \mathrm{m}), 5.11$ $(1 \mathrm{H}, \mathrm{m}), 5.08(1 \mathrm{H}, \mathrm{m}), 3.26(2 \mathrm{H}, \mathrm{m}), 1.70(2 \mathrm{H}, \mathrm{m}), 1.36(3 \mathrm{H}, \mathrm{s}), 1.35(3 \mathrm{H}, \mathrm{s}), 1.34$ $(3 \mathrm{H}, \mathrm{s}), 1.34(3 \mathrm{H}, \mathrm{s}) .{ }^{13} \mathrm{C} \mathrm{NMR}\left(125 \mathrm{MHz}, \mathrm{CDCl}_{3}\right) \delta$ 142.7, 140.6, 131.9, 115.2, 
74.5, 71.9, 31.6, 31.6, 30.1. ${ }^{\mathrm{S}} \mathrm{IR}$ (thin film NaCl) 3078, 2935, 1630, 1385, 1366, $1180 \mathrm{~cm}^{-1}$. HRMS ESI $(\mathrm{m} / \mathrm{z})$ : $[\mathrm{M}+\mathrm{Na}]^{+}$calcd for $\mathrm{C}_{11} \mathrm{H}_{20} \mathrm{O}_{2} \mathrm{Na}, 207.1361$; found, 207.1357.

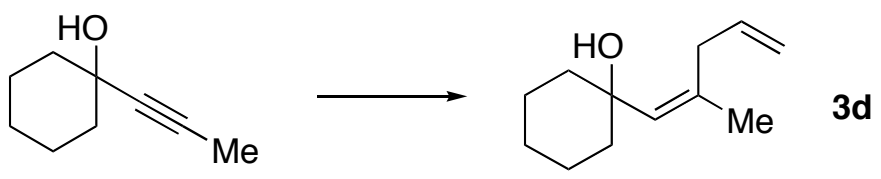

(Z)-1-(2-Methylpenta-1,4-dienyl)cyclohexanol (3d). Reaction was conducted with 1-(1-propynyl)cyclohexanol (38 mg, $0.273 \mathrm{mmol}$ ) and allyl bromide $(71 \mu \mathrm{L}$, $0.819 \mathrm{mmol}, 300 \mathrm{~mol} \%)$, using standard $\mathrm{Cul} \cdot \mathrm{P}(\mathrm{OEt})_{3}$ or $\mathrm{CuCl}$ procedure. Purification by flash chromatography (silica; $3 \% \mathrm{EtOAc/hex}$ ) provided the title compound ( $96 \%$ and $95 \%$ yield, respectively). $\mathrm{R}_{f} 0.40$ (10\% EtOAc/hex), no UV, purple (vanillin). ${ }^{1} \mathrm{H} \mathrm{NMR}\left(500 \mathrm{MHz}, \mathrm{CDCl}_{3}\right) \delta 5.84(1 \mathrm{H}$, ddt, $J=6.3,10.1,17.1)$, $5.39(1 \mathrm{H}, \mathrm{s}), 5.07(1 \mathrm{H}, \mathrm{dd}, J=1.8,17.1), 5.03(1 \mathrm{H}, \mathrm{dd}, J=1.8,10.1), 3.14(2 \mathrm{H}, \mathrm{d}$, $J=6.3), 1.72(3 \mathrm{H}, \mathrm{s}), 1.62(4 \mathrm{H}, \mathrm{m}), 1.45(4 \mathrm{H}, \mathrm{m}), 1.35(2 \mathrm{H}, \mathrm{m}) .{ }^{13} \mathrm{C}$ NMR $\left(125 \mathrm{MHz}, \mathrm{CDCl}_{3}\right) \delta 137.2,136.3,133.0,115.4,72.1,39.9,37.3,25.6,24.8,22.7$. IR (thin film NaCl) 3040, 2920, 1625, $1170 \mathrm{~cm}^{-1}$. HRMS ESI $(\mathrm{m} / \mathrm{z}):[\mathrm{M}+\mathrm{Na}]^{+}$calcd for $\mathrm{C}_{12} \mathrm{H}_{20} \mathrm{ONa}$, 203.1412; found, 203.1414.

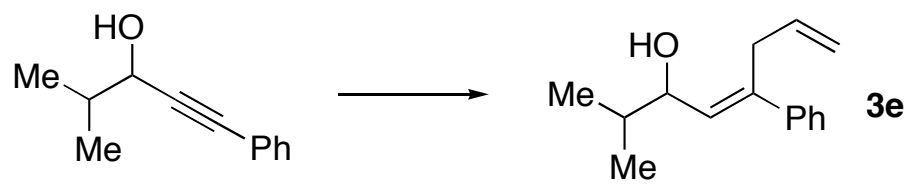

(E)-2-Methyl-5-phenylocta-4,7-dien-3-ol (3e). Reaction was conducted with 1phenyl-4-methyl-1-pentyn-3-ol (48 $\mu \mathrm{L}, 0.273 \mathrm{mmol})$ and allyl bromide $(71 \mu \mathrm{L}$, $0.819 \mathrm{mmol}, 300 \mathrm{~mol} \%)$, using standard $\mathrm{Cul} \cdot \mathrm{P}(\mathrm{OEt})_{3}$ or $\mathrm{CuCl}$ procedure. Purification by flash chromatography (silica; $\underline{5}, \underline{7} \%$ EtOAc/hex) provided the title compound ( $81 \%$ and $89 \%$ yield, respectively). $R_{f} 0.40$ (20\% EtOAc/hex), UV active, blue (vanillin). ${ }^{1} \mathrm{H} \mathrm{NMR}\left(500 \mathrm{MHz}, \mathrm{CDCl}_{3}\right)$ \& 7.40-7.42 (2H, m), 7.31-7.35 $(3 \mathrm{H}, \mathrm{m}), 5.82-5.90(2 \mathrm{H}, \mathrm{m}), 5.10(1 \mathrm{H}, \mathrm{ddt}, J=1.7,3.6,17.1), 5.03(1 \mathrm{H}$, ddt,

(S7) In compound $3 \mathbf{c}$, two ${ }^{13} \mathrm{C}$ resonances, corresponding to two sets of equivalent methyl groups, appear as a peak with a large shoulder, at 31.611 and $31.593 \mathrm{ppm}$, respectively. 
$J=1.7,3.4,10.2), 4.22(1 \mathrm{H}, \mathrm{dd}, J=7.8,7.9), 3.35(2 \mathrm{H}, \mathrm{m}), 1.81(1 \mathrm{H}, \mathrm{dtt}, J=7.8$, 6.7, 6.9), $1.48(1 \mathrm{H}, \mathrm{bs}), 1.04(3 \mathrm{H}, \mathrm{d}, J=6.7), 0.96(3 \mathrm{H}, \mathrm{d}, J=6.9) .{ }^{13} \mathrm{C}$ NMR $\left(125 \mathrm{MHz}, \mathrm{CDCl}_{3}\right) \delta 142.4,140.5,136.5,131.0,128.5,127.5,126.6,116.1,73.9$, 34.9, 34.7, 18.7, 18.5. IR (thin film NaCl) 3360, 3040, 2940, 1380, 1362, $870 \mathrm{~cm}^{-}$ 1. HRMS ESI $(\mathrm{m} / \mathrm{z})$ : $[\mathrm{M}+\mathrm{Na}]^{+}$calcd for $\mathrm{C}_{15} \mathrm{H}_{20} \mathrm{ONa}, 239.1412$; found, 239.1412.

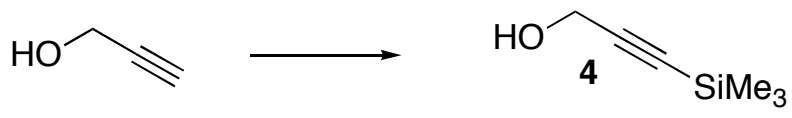

3-(Trimethylsilyl)prop-2-yn-1-ol (4). ${ }^{\mathrm{S}}$ A solution of propargyl alcohol $(1.00 \mathrm{~mL}$, $17.2 \mathrm{mmol})$ in THF $(43.0 \mathrm{~mL}, 0.400 \mathrm{M})$ was cooled to $-78^{\circ} \mathrm{C}$, and $n$-BuLi $(2.4 \mathrm{M}$ in hexanes, $21.5 \mathrm{~mL}, 51.5 \mathrm{mmol}, 300 \mathrm{~mol} \%$ ) was added dropwise. After stirring for $20 \mathrm{~min}$ at $-78{ }^{\circ} \mathrm{C}$, TMS-Cl $(7.70 \mathrm{~mL}, 60.1 \mathrm{mmol}, 350 \mathrm{~mol} \%)$ was added dropwise. The reaction was stirred for $5 \mathrm{~min}$ at $-78^{\circ} \mathrm{C}$, the cold bath was removed, and the reaction stirred $1 \mathrm{~h}$. The reaction was quenched with $50 \mathrm{~mL}$ water and then $10 \% \mathrm{HCl}$ was added to the crude reaction mixture until complete consumption of the TMS-ether was observed (TLC analysis, $R_{f} 0.90(10 \%$ EtOAc/hex), no UV, purple (vanillin)). The reaction was poured into a separatory funnel containing $100 \mathrm{~mL} \mathrm{Et}_{2} \mathrm{O}$, shaken, and the layers were separated. The aqueous layer was extracted $2 \times$ into $100 \mathrm{~mL} \mathrm{Et}_{2} \mathrm{O}$. The combined organic layers were washed with brine, dried over $\mathrm{MgSO}_{4}$, filtered, and carefully concentrated in vacuo $\left(10\right.$ torr, $0^{\circ} \mathrm{C}$ ). Purification by flash chromatography (silica; $5, \underline{20} \%$ $\mathrm{Et}_{2} \mathrm{O}$ /pent) provided the TMS-propargyl alcohol $(2.15 \mathrm{~g}, 97.6 \%)$ as a pale yellow oil. $\mathrm{R}_{f} 0.25$ (10\% EtOAc/hex), no UV, purple (vanillin). ${ }^{1} \mathrm{H} \mathrm{NMR}\left(500 \mathrm{MHz}, \mathrm{CDCl}_{3}\right)$ $\delta 4.28(2 \mathrm{H}, \mathrm{s}), 1.62(1 \mathrm{H}, \mathrm{bs}), 0.19(9 \mathrm{H}, \mathrm{s}) .{ }^{13} \mathrm{C} \mathrm{NMR}\left(125 \mathrm{MHz}, \mathrm{CDCl}_{3}\right) \delta 104.2$, 90.5, 51.5, -0.1. IR (thin film NaCl) $3430,2200,1036 \mathrm{~cm}^{-1}$. HRMS ESI $(\mathrm{m} / z)$ : $[\mathrm{M}+\mathrm{Na}]^{+}$calcd for $\mathrm{C}_{6} \mathrm{H}_{12} \mathrm{OSiNa}, 151.0555$; found, 151.0553 .

(S8) Procedure amended from: (a) Velcicky, J.; Lanver, A.; Lex, J.; Prokop, A.; Wieder, T.; Schmalz, H.-G. Chem. Eur. J. 2004, 10, 5087-5110. (b) Jones, T. K.; Denmark, S. E. Org. Synth. 1986, 64, 182-188. 


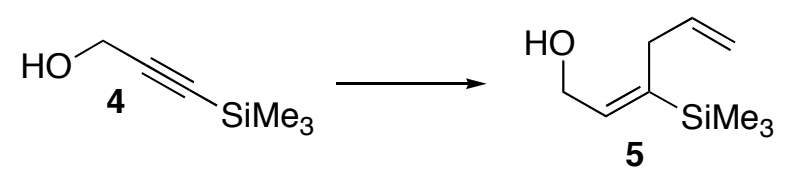

(E)-3-(trimethylsilyl)hexa-2,5-dien-1-ol (5). Reaction was conducted with alcohol 4 (45 mg, $0.273 \mathrm{mmol})$ and allyl bromide $(71 \mu \mathrm{L}, 0.819 \mathrm{mmol}, 300 \mathrm{~mol}$ $\%)$, using standard Cul· $P(\mathrm{OEt})_{3}$ procedure. Purification by flash chromatography (silica; 5, 10\% EtOAc/hex) provided the title compound (44.3 mg, 95\%). $\mathrm{R}_{f} 0.50$ (20\% EtOAc/hex), no UV, blue (vanillin). ${ }^{1} \mathrm{H} \mathrm{NMR}\left(500 \mathrm{MHz}, \mathrm{CDCl}_{3}\right) \delta 6.02(1 \mathrm{H}$, t, $J=6.0), 5.75(1 \mathrm{H}, \mathrm{m}), 5.00(1 \mathrm{H}, \mathrm{m}), 4.97(1 \mathrm{H}, \mathrm{m}), 4.25(2 \mathrm{H}, \mathrm{d}, J=6.0), 2.93$ $(2 \mathrm{H}, \mathrm{d}, J=6.1), 1.55(1 \mathrm{H}, \mathrm{bs}), 0.09(9 \mathrm{H}, \mathrm{s}) .{ }^{13} \mathrm{C} \mathrm{NMR}\left(125 \mathrm{MHz}, \mathrm{CDCl}_{3}\right) \delta 141.2$, 140.0, 137.0, 115.0, 59.9, 34.0, -1.4. IR (thin film NaCl) 3462, 3075, 2920, $1646 \mathrm{~cm}^{-1}$. HRMS ESI $(\mathrm{m} / z)$ : $[\mathrm{M}+\mathrm{Na}]^{+}$calcd for $\mathrm{C}_{9} \mathrm{H}_{18} \mathrm{OSiNa}, 193.1025$; found, 193.1027.

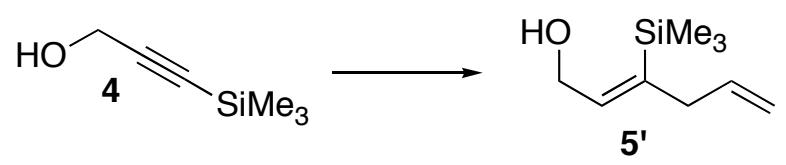

(Z)-3-(Trimethylsilyl)hexa-2,5-dien-1-ol (5'). Following Ziegler's procedure, ${ }^{\mathrm{s9}} \mathrm{a}$ round-bottom flask was charged with alcohol $4(35 \mathrm{mg}, 0.273 \mathrm{mmol})$ and $\mathrm{Et}_{2} \mathrm{O}$ $(0.55 \mathrm{~mL}, 0.50 \mathrm{M})$, and the resulting solution was cooled to $-20^{\circ} \mathrm{C}$. Neat i- $\mathrm{Bu}_{2} \mathrm{AlH}(0.14 \mathrm{~mL}, 0.955 \mathrm{mmol}, 350 \mathrm{~mol} \%)$ was added dropwise, and after 2 min, the cold bath was removed. The reaction was heated to gentle reflux $\left(40{ }^{\circ} \mathrm{C}\right)$. After $12 \mathrm{~h}$, the reaction was cooled to $0{ }^{\circ} \mathrm{C}$, then MeLi $\left(1.6 \mathrm{M}\right.$ in $\mathrm{Et}_{2} \mathrm{O}$, $0.64 \mathrm{~mL}, 1.02 \mathrm{mmol}, 375 \mathrm{~mol} \%$ ) was added dropwise. The cold bath was removed and, after stirring for $90 \mathrm{~min}$, the reaction was cooled to $-78{ }^{\circ} \mathrm{C}$ $\left(\mathrm{CO}_{2} /\right.$ acetone $)$. A solution of Cul.P(OEt $)_{3}(86 \mathrm{mg}, 100 \mathrm{~mol} \%)$ in THF $(1.4 \mathrm{~mL}$, $0.20 \mathrm{M}$ ) was added by cannula (exothermic!), followed by addition of neat allyl bromide $(71 \mu \mathrm{L}, 0.819 \mathrm{mmol}, 300 \mathrm{~mol} \%)$. The reaction was stirred for $24 \mathrm{~h}$, allowing the cold bath to warm slowly to rt. The crude reaction mixture was

(S9) Ziegler, F. E.; Mikami, K. Tetrahedron Lett. 1984, 25, 131-134. 
poured onto sat. aq. potassium sodium tartrate $(30 \mathrm{~mL})$, rinsed with $30 \mathrm{~mL}$ EtOAc, and the mixture was stirred for $30 \mathrm{~min}$. The layers were separated, and the aqueous layer was extracted $2 x$ into $30 \mathrm{~mL}$ EtOAc. The combined organic layers were washed with brine, dried over $\mathrm{MgSO}_{4}$, filtered, and concentrated in vacuo. Purification by flash chromatography (silica; $5,10 \%$ EtOAc/hex) provided the title compound ( $20.0 \mathrm{mg}, 43 \%)$. $\mathrm{R}_{f} 0.50$ (20\% EtOAc/hex), no UV, blue (vanillin). ${ }^{1} \mathrm{H} \mathrm{NMR}\left(500 \mathrm{MHz}, \mathrm{CDCl}_{3}\right) \delta 6.15(1 \mathrm{H}, \mathrm{t}, J=6.9), 5.81(1 \mathrm{H}, \mathrm{m}), 5.03$ $(1 \mathrm{H}, \mathrm{m}), 5.01(1 \mathrm{H}, \mathrm{m}), 4.24(2 \mathrm{H}, \mathrm{d}, J=7.1), 2.87(2 \mathrm{H}, \mathrm{d}, J=6.4), 1.54(1 \mathrm{H}, \mathrm{bs})$, $0.17(9 \mathrm{H}, \mathrm{s}) .{ }^{13} \mathrm{C}$ NMR $\left(125 \mathrm{MHz}, \mathrm{CDCl}_{3}\right) \delta 140.8,138.6,137.8,115.8,59.2$, 40.6, -1.2. IR (thin film $\mathrm{NaCl}$ ) 3470, 3090, 2942, $1660 \mathrm{~cm}^{-1}$. HRMS ESI $(\mathrm{m} / z)$ : $[\mathrm{M}+\mathrm{Na}]^{+}$calcd for $\mathrm{C}_{9} \mathrm{H}_{18} \mathrm{OSiNa}, 193.1025$; found, 193.1024.

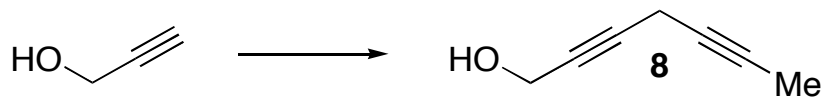

Hepta-2,5-diyn-1-ol (8). ${ }^{\mathrm{S} 10}$ To a rt solution of EtMgCl (2.0 M in THF, $18.0 \mathrm{~mL}$, $36.0 \mathrm{mmol}, 284 \mathrm{~mol} \%$ ) was added by cannula, portionwise over $25 \mathrm{~min}$, a solution of propargyl alcohol $(1.01 \mathrm{~mL}, 17.1 \mathrm{mmol}, 135 \mathrm{~mol} \%)$ in THF $(2.20 \mathrm{~mL}$, $5.8 \mathrm{M}$ ). Once the addition was complete, the reaction was heated to reflux for $90 \mathrm{~min}$. The flask was cooled to $0^{\circ} \mathrm{C}$, then solid $\mathrm{CuCl}(126 \mathrm{mg}, 1.27 \mathrm{mmol}$, $10.0 \mathrm{~mol} \%)$ was added. After stirring for $15 \mathrm{~min}$ at $0{ }^{\circ} \mathrm{C}$, 1-bromo-2-butyne (1.14 mL, $12.7 \mathrm{mmol}$ ) was added dropwise over $10 \mathrm{~min}$. Upon complete addition, the reaction was heated to reflux for $16 \mathrm{~h}$. The reaction was flask was cooled to rt, then quenched by careful addition, over $10 \mathrm{~min}$, of $87 \mathrm{~mL} 10 \%$ aq. $\mathrm{NH}_{4} \mathrm{Cl}$ (exothermic!). The mixture was added to a separatory funnel containing $80 \mathrm{~mL}$ $\mathrm{Et}_{2} \mathrm{O}$, and the layers were separated. The aqueous layer was extracted $2 \times$ into $80 \mathrm{~mL} \mathrm{Et} \mathrm{t}_{2} \mathrm{O}$. The combined organic layers were washed with brine, dried over $\mathrm{MgSO}_{4}$, filtered, and carefully concentrated in vacuo $\left(10\right.$ torr, $\left.0^{\circ} \mathrm{C}\right)$. Distillation

(S10) Bushby, R. J.; Whitham, G. H. J. Chem. Soc. B 1970, 563-569. 
(5 torr, $150^{\circ} \mathrm{C}$ ) provided the title compound $\left(997 \mathrm{mg}, 73 \%\right.$ ) as a colorless oil. ${ }^{\mathrm{S} 11}$ ${ }^{1} \mathrm{H} \mathrm{NMR}\left(500 \mathrm{MHz}, \mathrm{CDCl}_{3}\right) \delta 4.28(2 \mathrm{H}, \mathrm{m}), 3.17(2 \mathrm{H}, \mathrm{q}, J=2.5), 1.81(3 \mathrm{H}, \mathrm{t}$, $J=2.5), 1.59(1 \mathrm{H}, \mathrm{bs})$.

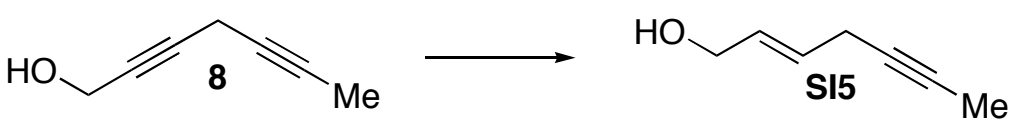

(E)-Hept-2-en-5-yn-1-ol (SI5). ${ }^{\text {s12 }}$ To a $0{ }^{\circ} \mathrm{C}$ solution of diynol 8 (250 mg, $2.31 \mathrm{mmol}, 105 \mathrm{~mol} \%)$ in $\mathrm{Et}_{2} \mathrm{O}(4.62 \mathrm{~mL}, 0.50 \mathrm{M})$ was added, slowly over $10 \mathrm{~min}$, solid $\mathrm{LiAlH}_{4}(84.0 \mathrm{mg}, 2.20 \mathrm{mmol})$. Upon complete addition, the reaction was heated to a gentle reflux for $7 \mathrm{~h}$. To the crude mixture were carefully added in sequence: $3.0 \mathrm{~mL} \mathrm{Et} \mathrm{t}_{2} \mathrm{O}, 84 \mu \mathrm{L} \mathrm{H}_{2} \mathrm{O}, 84 \mu \mathrm{L}$ of $2 \mathrm{~N}$ aq. $\mathrm{NaOH}$, and then $0.24 \mathrm{~mL}$ $\mathrm{H}_{2} \mathrm{O}$. A small amount of $\mathrm{MgSO}_{4}$ was added to the flask, and the resulting mixture was stirred at $\mathrm{rt}$ for $5 \mathrm{~min}$. After filtration through Celite, the residue was carefully concentrated in vacuo $\left(10\right.$ torr, $0^{\circ} \mathrm{C}$ ) to afford the title compound ( $258 \mathrm{mg}, 106 \%$ theoretical yield) as a colorless oil. The crude product was used without further purification. $\mathrm{R}_{f} 0.10$ (5\% EtOAc/hex), no UV, blue-green (vanillin). ${ }^{1} \mathrm{H} N \mathrm{NR}$ $\left(500 \mathrm{MHz}, \mathrm{CDCl}_{3}\right) \delta 5.92(1 \mathrm{H}, \mathrm{m}), 5.71(1 \mathrm{H}, \mathrm{m}), 4.15(2 \mathrm{H}, \mathrm{dd}, J=1.0,5.7), 2.93$ $(2 \mathrm{H}, \mathrm{m}), 1.83(3 \mathrm{H}, \mathrm{t}, J=2.5), 1.46(1 \mathrm{H}, \mathrm{bs})$.

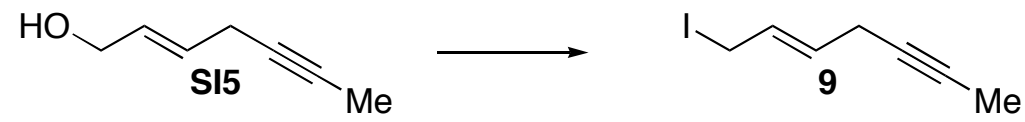

(E)-1-lodohept-2-en-5-yne (9). To a $0{ }^{\circ} \mathrm{C}$ solution of $\mathrm{PPh}_{3}(131 \mathrm{mg}, 0.499 \mathrm{mmol}$, $110 \mathrm{~mol} \%$ ) in $1.30 \mathrm{~mL} \mathrm{CH}_{2} \mathrm{Cl}_{2}$ were added sequentially: imidazole (43.0 mg, $0.636 \mathrm{mmol}, 140 \mathrm{~mol} \%)$, iodine (138 $\mathrm{mg}, 0.545 \mathrm{mmol}, 120 \mathrm{~mol} \%)$, and a solution of crude alcohol SI5 (50.0 mg, $0.454 \mathrm{mmol})$ in $\mathrm{CH}_{2} \mathrm{Cl}_{2}(1.00 \mathrm{~mL}, 0.200 \mathrm{M}$ total). The reaction was stirred at $0{ }^{\circ} \mathrm{C}$, protected from light, for $30 \mathrm{~min}$. The reaction was quenched by addition of $40 \mathrm{~mL}$ of $20 \%$ aq. $\mathrm{Na}_{2} \mathrm{~S}_{2} \mathrm{O}_{3}$. The aqueous

(S11) Upon storage ( 2 days, $4^{\circ} \mathrm{C}$ ), a yellow color begins to evolve. Optimum yield in subsequent steps was obtained when diynol 8 was colorless (i.e. freshly distilled, or frozen at $-10^{\circ} \mathrm{C}$ ).

(S12) Aebi, J. D.; Deyo, D. T.; Sun, G. C.; Guillaume, D.; Dunlap, B.; Rich, D. H. J. Med. Chem. 1990, 33, 999-1009. 
layer was extracted $3 \times$ into $50 \mathrm{~mL} \mathrm{CH}_{2} \mathrm{Cl}_{2}$. The combined organic layers were washed with brine, dried over $\mathrm{MgSO}_{4}$, filtered, and carefully concentrated in vacuo $\left(10\right.$ torr, $0^{\circ} \mathrm{C}$ ). Purification by flash chromatography (silica; $\underline{0}, \underline{2} \%$ $\mathrm{Et}_{2} \mathrm{O}$ /pent) provided the title compound (93 $\mathrm{mg}, 93 \%$ ) as a faint pink oil. $\mathrm{R}_{f} 0.60$ (5\% EtOAc/hex), UV active, green (vanillin). ${ }^{1} \mathrm{H} \mathrm{NMR}\left(500 \mathrm{MHz}, \mathrm{CDCl}_{3}\right) \delta 6.00$ $(1 \mathrm{H}, \mathrm{dt}, J=8.0,15.0), 5.70(1 \mathrm{H}, \mathrm{dt}, J=5.4,15.0), 3.90(2 \mathrm{H}, \mathrm{d}, J=8.0), 2.91(2 \mathrm{H}$, $\mathrm{dq}, J=2.5,5.4), 1.83(3 \mathrm{H}, \mathrm{t}, J=2.5)$. The iodide proved somewhat unstable, and was utilized immediately in subsequent reactions.
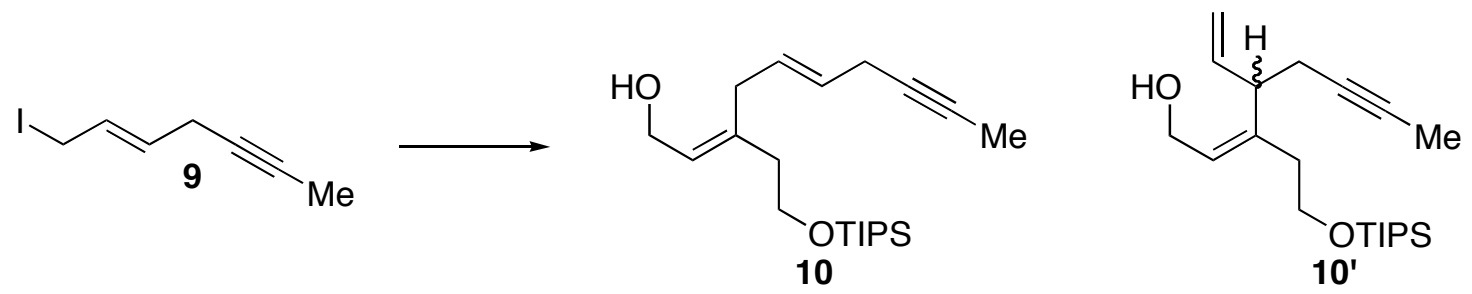

(2E,5E)-3-(2-(Triisopropylsilyloxy)ethyl)deca-2,5-dien-8-yn-1-ol

(10) and (Z)-3-(2-(Triisopropylsilyloxy)ethyl)-4-vinyloct-2-en-6-yn-1-ol (10').

Reaction was conducted with alcohol 1 (100 mg, $0.390 \mathrm{mmol})$ and allyl iodide 9 (257 mg, $1.17 \mathrm{mmol}, 300 \mathrm{~mol} \%)$, using standard $\mathrm{CuCl}$ procedure. Purification by flash chromatography (silica; $\underline{6} \%$ EtOAc/hex) initially provided the $\mathrm{S}_{\mathrm{N}} 2^{\prime}$ product ${ }^{\prime} \mathrm{\prime}^{\prime}(62.9 \mathrm{mg}, 46.0 \%)$ as a colorless oil. Further elution from the column (silica; $\underline{6}, \underline{8} \%$ EtOAc/hex) provided the $\mathrm{S}_{\mathrm{N}} 2$ product 10 as a pale yellow oil (69.7 mg, 51.0\%).

- Compound 10. $R_{f} 0.30$ (20\% EtOAc/hex), no UV, green (vanillin). ${ }^{1} \mathrm{H}$ NMR $\left(500 \mathrm{MHz}, \mathrm{CDCl}_{3}\right) \delta 5.62(1 \mathrm{H}, \mathrm{m}), 5.52(1 \mathrm{H}, \mathrm{t}, J=6.9), 5.45(1 \mathrm{H}, \mathrm{m}), 4.17(2 \mathrm{H}, \mathrm{d}$, $J=6.9), 3.77(2 \mathrm{H}, \mathrm{t}, J=7.1), 2.86(2 \mathrm{H}, \mathrm{m}), 2.84(2 \mathrm{H}, \mathrm{d}, J=7.2), 2.29(2 \mathrm{H}, \mathrm{t}$, $J=7.1), 1.81(3 \mathrm{H}, \mathrm{t}, J=2.5) .{ }^{13} \mathrm{C} \mathrm{NMR}\left(125 \mathrm{MHz}, \mathrm{CDCl}_{3}\right) \delta 139.2,131.0,129.0$, 126.4, 80.3, 76.5, 62.7, 59.4, 40.5, 34.0, 22.1, 18.2, 12.2, 3.7. IR (thin film NaCl) 3465, 3040, 2926, 2270,1240, $1060 \mathrm{~cm}^{-1}$. HRMS ESI $(\mathrm{m} / \mathrm{z}):[\mathrm{M}+\mathrm{Na}]^{+}$calcd for $\mathrm{C}_{21} \mathrm{H}_{38} \mathrm{O}_{2} \mathrm{SiNa}$, 373.2539; found, 373.2537. 
- Compound 10'. $\mathrm{R}_{f} 0.40$ (20\% EtOAc/hex), no UV, brown (vanillin). ${ }^{1} \mathrm{H}$ NMR $\left(500 \mathrm{MHz}, \mathrm{CDCl}_{3}\right) \delta 5.83(1 \mathrm{H}, \mathrm{ddd}, J=6.3,10.5,17.2), 5.66(1 \mathrm{H}, \mathrm{t}, J=7.2), 5.09$ $(1 \mathrm{H}, \mathrm{ddd}, J=1.3,1.4,10.5), 5.07(1 \mathrm{H}, \mathrm{ddd}, J=1.4,1.5,17.2), 4.27(1 \mathrm{H}, \mathrm{dd}$, $J=7.6,12.3), 4.12(1 \mathrm{H}, \mathrm{dd}, J=6.9,12.3), 3.78(2 \mathrm{H}, \mathrm{t}, J=7.2), 3.45(1 \mathrm{H}, \mathrm{m})$, $2.36(2 \mathrm{H}, \mathrm{m}), 2.24(2 \mathrm{H}, \mathrm{t}, J=7.6), 1.77(3 \mathrm{H}, \mathrm{t}, J=2.5), 1.44(1 \mathrm{H}, \mathrm{bs}), 1.06-1.07$ $(21 \mathrm{H}, \mathrm{m}) .{ }^{13} \mathrm{C}$ NMR $\left(125 \mathrm{MHz}, \mathrm{CDCl}_{3}\right) \delta 140.7,138.9,127.3,115.6,77.9,77.4$, 63.5, 58.6, 44.2, 35.3, 22.5, 18.2, 12.2, 3.6. IR (thin film NaCl) 3480, 3026, 2909, 2265, 1648, $1025 \mathrm{~cm}^{-1}$. HRMS ESI $(\mathrm{m} / z):[\mathrm{M}+\mathrm{Na}]^{+}$calcd for $\mathrm{C}_{21} \mathrm{H}_{38} \mathrm{O}_{2} \mathrm{SiNa}$, 373.2539; found, 373.2547 .
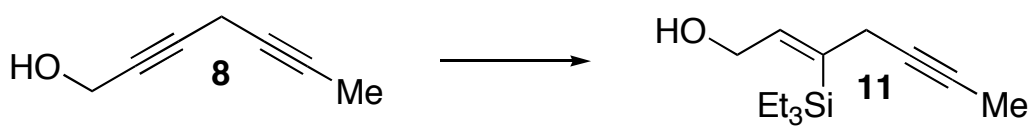

(Z)-3-(Triethylsilyl)hept-2-en-5-yn-1-ol (11). To a $0{ }^{\circ} \mathrm{C}$ solution of distilled diyne 8 (50 mg, $0.46 \mathrm{mmol})$ in $\mathrm{CH}_{2} \mathrm{Cl}_{2}(0.93 \mathrm{~mL}, 0.50 \mathrm{M})$ was added $\mathrm{Et}_{3} \mathrm{SiH}(92 \mu \mathrm{L}$, $0.575 \mathrm{mmol}, 123 \mathrm{~mol} \%$ ). Solid [Cp* $\left.\mathrm{Ru}(\mathrm{MeCN})_{3}\right] \mathrm{PF}_{6}{ }^{\mathrm{S} 13, S 14}$ (3.5 mg, $6.94 \mu \mathrm{mol}$, $1.5 \mathrm{~mol} \%$ ) was added, and the reaction was stirred for $20 \mathrm{~min}$. A second portion of $\mathrm{Ru}$ catalyst $(3.5 \mathrm{mg})$ was added, and the reaction was stirred an additional $30 \mathrm{~min}$. The reaction mixture was filtered through a $1 / 2 \mathrm{in}$. pad of $\mathrm{SiO}_{2}$, rinsed with $\mathrm{CH}_{2} \mathrm{Cl}_{2}$, and concentrated in vacuo. Purification by flash chromatography (silica; 5, 10\% EtOAc/hex) provided the title compound (45 mg, 43\%). $\quad R_{f} 0.30$ (20\% EtOAc/hex), no UV, green (vanillin). ${ }^{1} \mathrm{H} N M R(500 \mathrm{MHz}$, $\left.\mathrm{CDCl}_{3}\right) \delta 6.57(1 \mathrm{H}, \mathrm{t}, J=7.0), 4.23(2 \mathrm{H}, \mathrm{d}, J=7.0), 2.94(2 \mathrm{H}, \mathrm{q}, J=2.5), 1.84$ $(3 \mathrm{H}, \mathrm{t}, J=2.5), 1.37(1 \mathrm{H}, \mathrm{bs}), 0.95(9 \mathrm{H}, \mathrm{t}, J=8.1), 0.70(6 \mathrm{H}, \mathrm{q}, J=8.1) .{ }^{13} \mathrm{C} \mathrm{NMR}$ $\left(125 \mathrm{MHz}, \mathrm{CDCl}_{3}\right) \delta 141.9,136.0,79.9,73.1,62.3,27.7,7.7,4.2,3.8$. IR (thin film NaCl) 3465, 3091, 2946, $2960 \mathrm{~cm}^{-1}$. HRMS ESI $(\mathrm{m} / z):[\mathrm{M}+\mathrm{Na}]^{+}$calcd for $\mathrm{C}_{13} \mathrm{H}_{24} \mathrm{OSiNa}$, 247.1494; found, 247.1497.

(S13) Tris(acetonitrile)pentamethylcyclopentadienylruthenium (I) hexafluorophosphate was purchased from Strem Chemicals and used without further purification.

(S14) Trost, B. M.; Ball, Z. T. J. Am. Chem. Soc. 2005, 127, 17644-17655. 


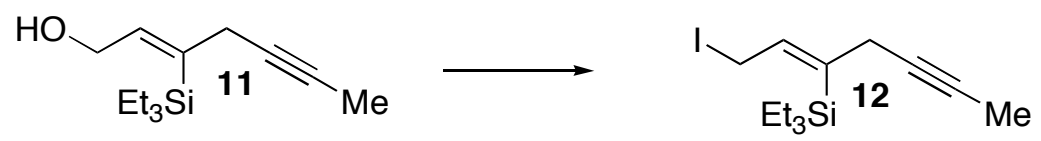

(Z)-Triethyl(1-iodohept-2-en-5-yn-3-yl)silane (12). To a $0{ }^{\circ} \mathrm{C}$ solution of $\mathrm{PPh}_{3}$

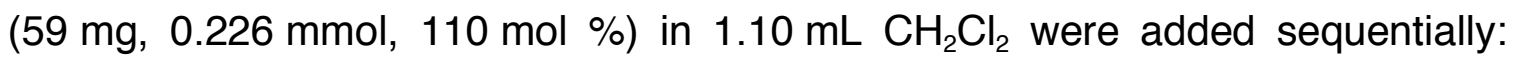
imidazole (20 mg, $0.287 \mathrm{mmol}, 140 \mathrm{~mol} \%$ ), iodine (62 mg, $0.246 \mathrm{mmol}, 120 \mathrm{~mol}$ $\%$ ), and a solution of alcohol $11(46 \mathrm{mg}, 0.205 \mathrm{mmol})$ in $\mathrm{CH}_{2} \mathrm{Cl}_{2}(1.00 \mathrm{~mL}$, $0.200 \mathrm{M}$ total). The reaction was stirred at $0{ }^{\circ} \mathrm{C}$, protected from light, for $60 \mathrm{~min}$. The reaction was quenched by addition of $40 \mathrm{~mL}$ of $20 \%$ aq. $\mathrm{Na}_{2} \mathrm{~S}_{2} \mathrm{O}_{3}$. The aqueous layer was extracted $3 \times$ into $50 \mathrm{~mL} \mathrm{CH}_{2} \mathrm{Cl}_{2}$. The combined organic layers were washed with brine, dried over $\mathrm{MgSO}_{4}$, filtered, and carefully concentrated in vacuo $\left(10\right.$ torr, $0^{\circ} \mathrm{C}$ ). Purification by flash chromatography (silica; $\underline{2}, \underline{4} \%$ $\mathrm{Et}_{2} \mathrm{O} /$ pent) provided the title compound $(91.5 \mathrm{mg}, 93 \%)$ as a faint pink oil. $\mathrm{R}_{f} 0.85$ (10\% EtOAc/hex), faint active, blue-green (vanillin). The iodide proved somewhat unstable, and was utilized immediately in subsequent reactions.

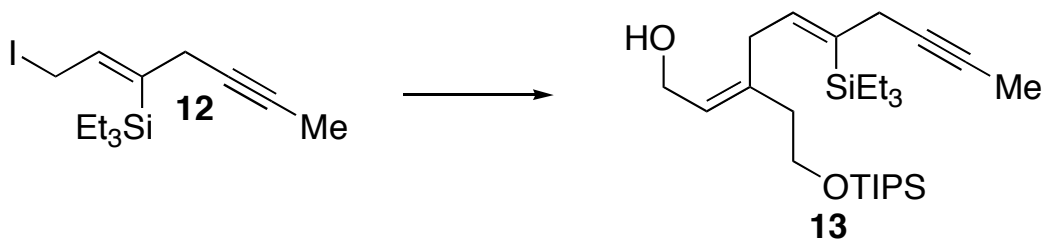

(2E,5Z)-6-(Triethylsilyl)-3-(2-(triisopropylsilyloxy)ethyl)deca-2,5-dien-8-yn-1ol (13). Reaction was conducted with alcohol $1(28 \mathrm{mg}, 0.109 \mathrm{mmol})$ and allyl iodide 12 (109 $\mathrm{mg}, 0.327 \mathrm{mmol}, 300 \mathrm{~mol} \%$ ), using standard $\mathrm{CuCl}$ procedure. Purification by flash chromatography (silica; $\underline{6} \% \mathrm{EtOAc/hex}$ ) provided the title compound (36.0 mg, 70.9\%) as a colorless oil. $R_{f} 0.50$ (20\% EtOAc/hex), faint active, blue (vanillin). ${ }^{1} \mathrm{H}$ NMR $\left(500 \mathrm{MHz}, \mathrm{CDCl}_{3}\right) \delta 6.17(1 \mathrm{H}, \mathrm{t}, J=6.5), 5.51(1 \mathrm{H}$, t, $J=7.0), 4.18(2 \mathrm{H}, \mathrm{d}, J=7.0), 3.78(2 \mathrm{H}, \mathrm{t}, J=7.0), 2.96(2 \mathrm{H}, \mathrm{d}, J=6.5), 2.88$ (2H, q, $J=2.0), 2.30$ (2H, t $J=7.0), 1.81(3 \mathrm{H}, \mathrm{t}, J=2.0), 1.41(1 \mathrm{H}, \mathrm{bs}), 1.05$ $1.06(21 \mathrm{H}, \mathrm{m}), 0.96(9 \mathrm{H}, \mathrm{t}, J=8.0), 0.73(6 \mathrm{H}, \mathrm{q}, J=8.0) .{ }^{13} \mathrm{C} N M R(125 \mathrm{MHz}$, $\left.\mathrm{CDCl}_{3}\right) \delta 141.9,139.9,133.5,126.2,79.1,77.7,62.6,59.4,40.5,33.5,27.9$, 18.2, 12.2, 7.7, 4.0, 3.8. IR (thin film NaCl) 3480, 3065, 2940, 2250, 1240, 
$1059 \mathrm{~cm}^{-1}$. HRMS ESI $(\mathrm{m} / \mathrm{z})$ : $[\mathrm{M}+\mathrm{Na}]^{+}$calcd for $\mathrm{C}_{27} \mathrm{H}_{52} \mathrm{O}_{2} \mathrm{Si}_{2} \mathrm{Na}$, 487.3404; found, 487.2404 . 


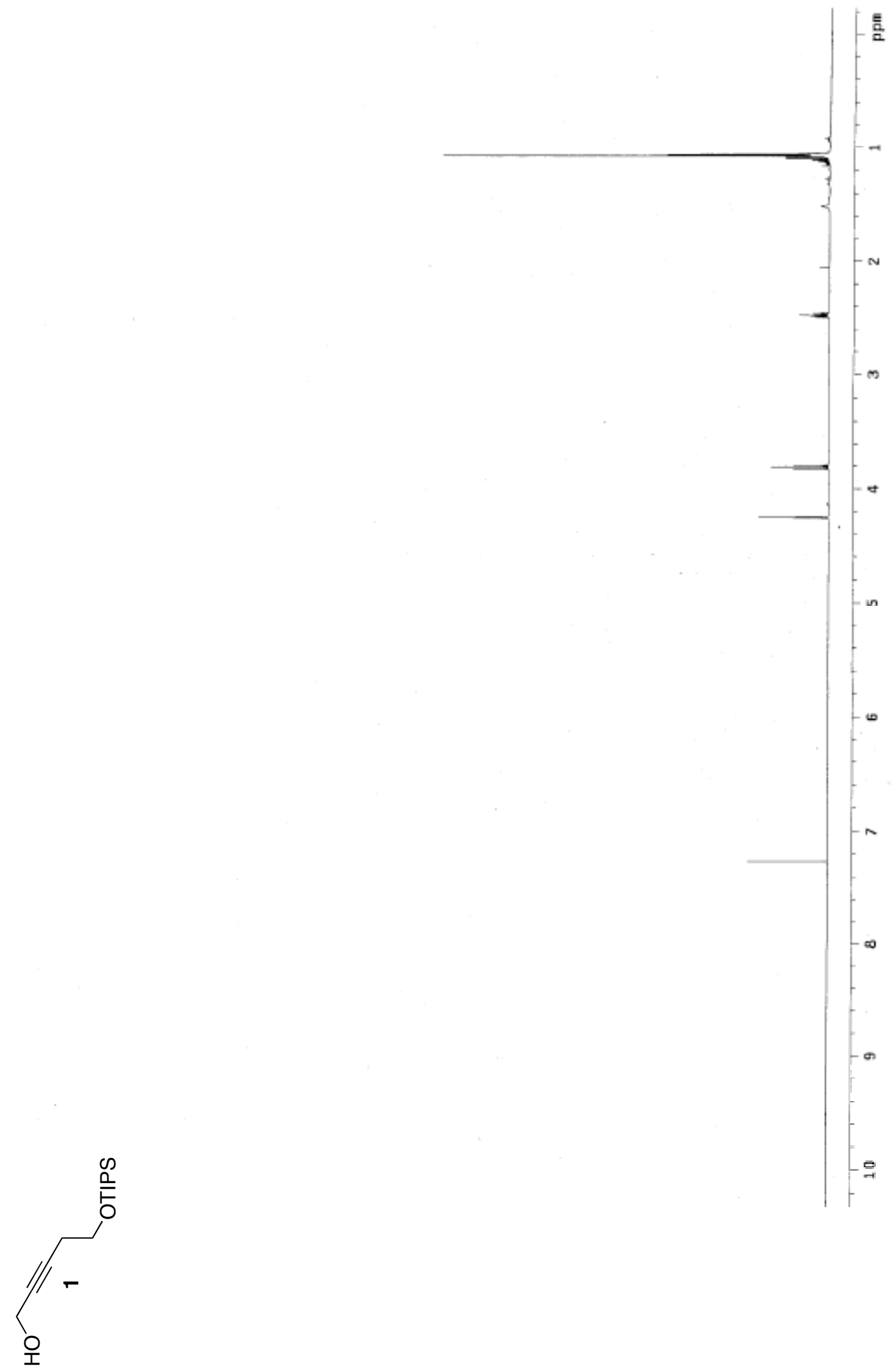

S-23 


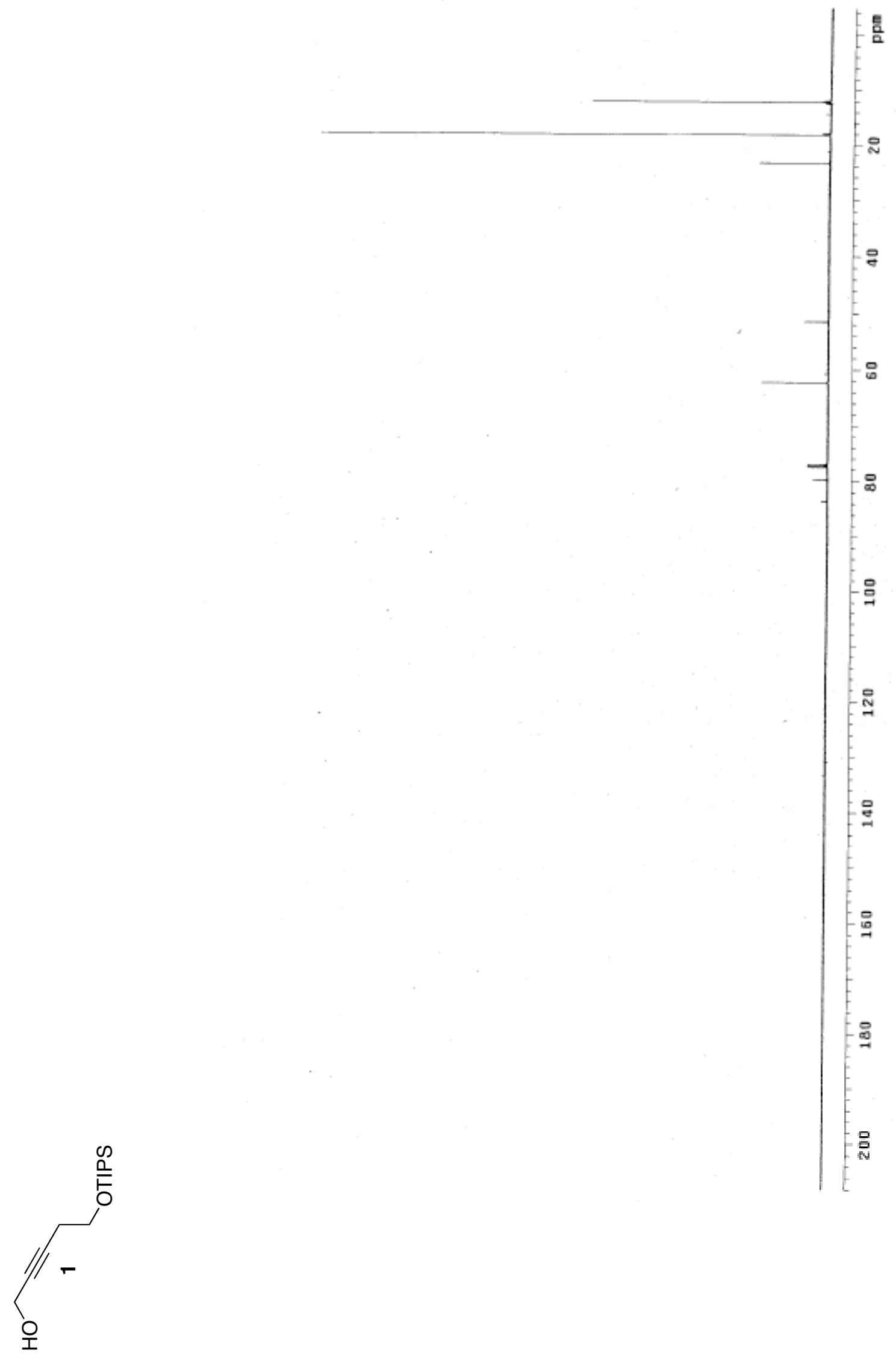

S-24 


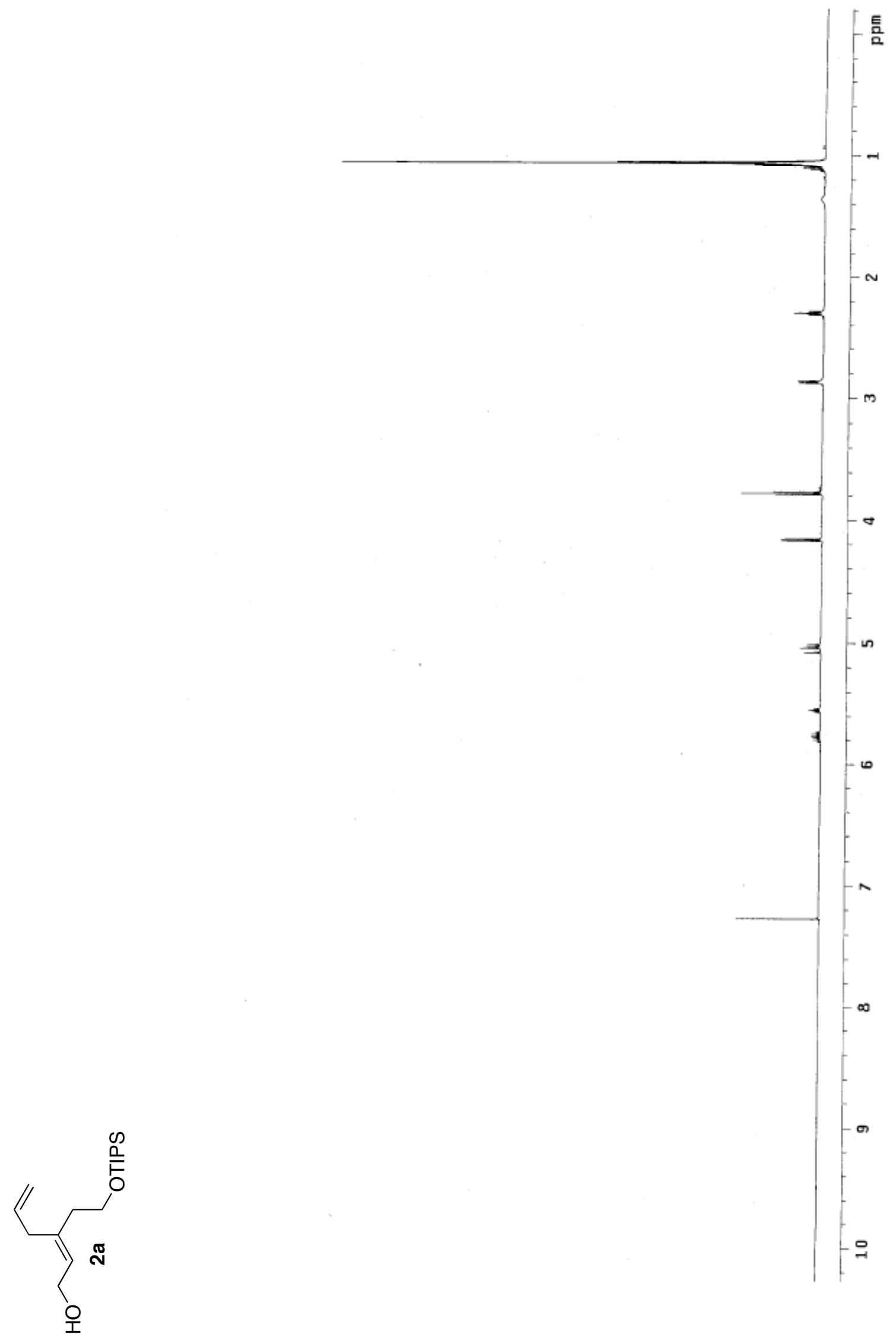

S-25 


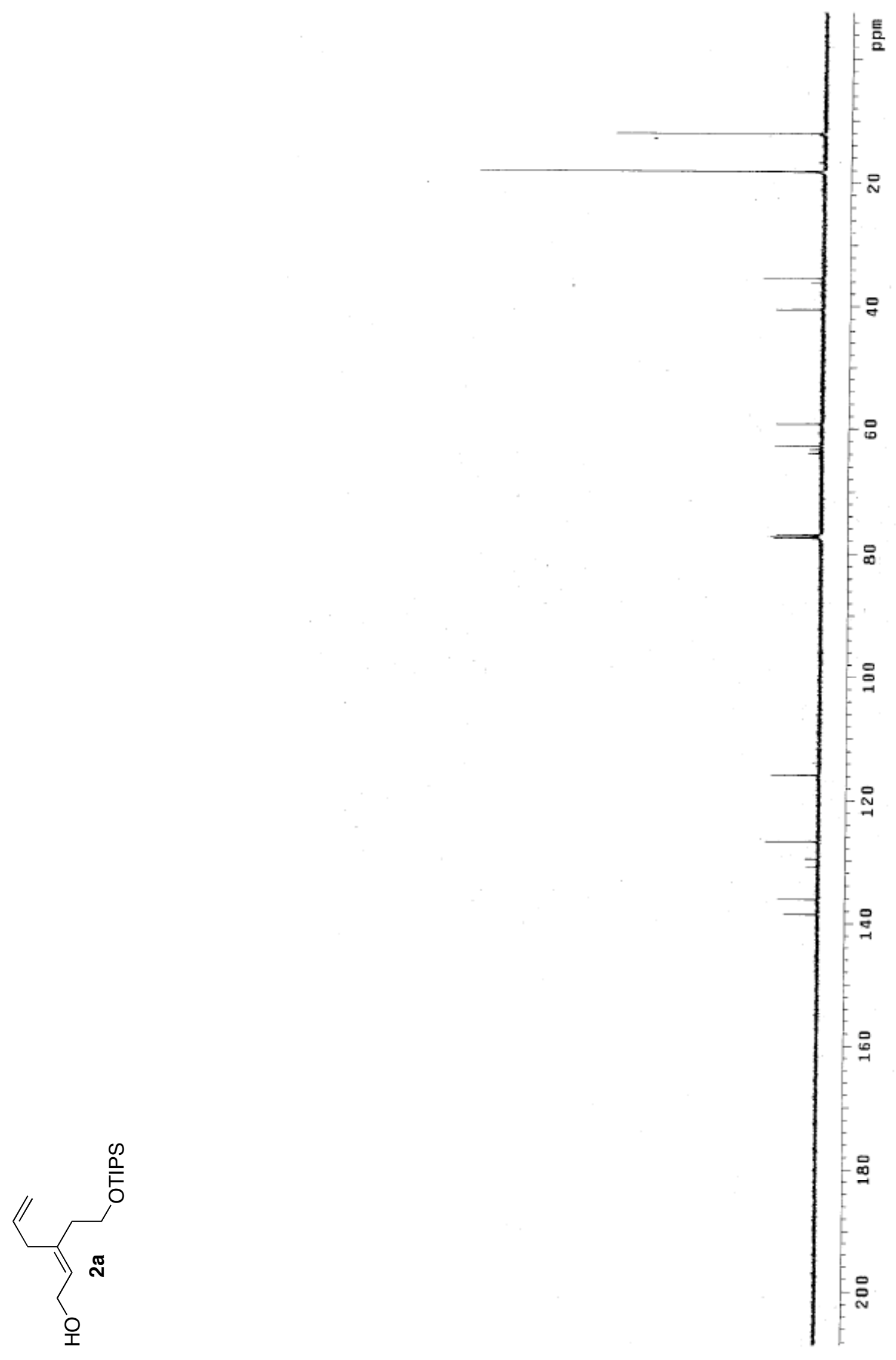




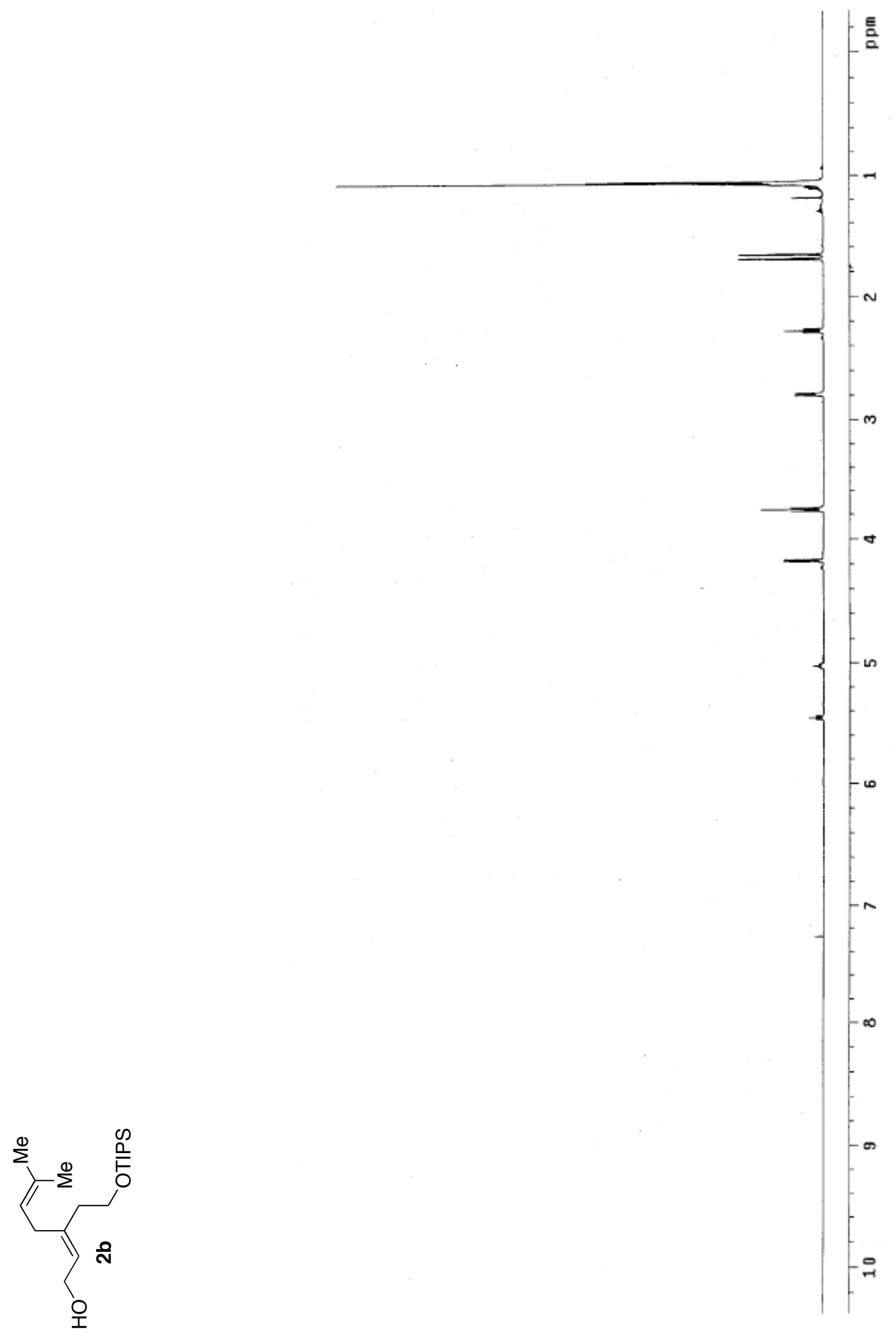




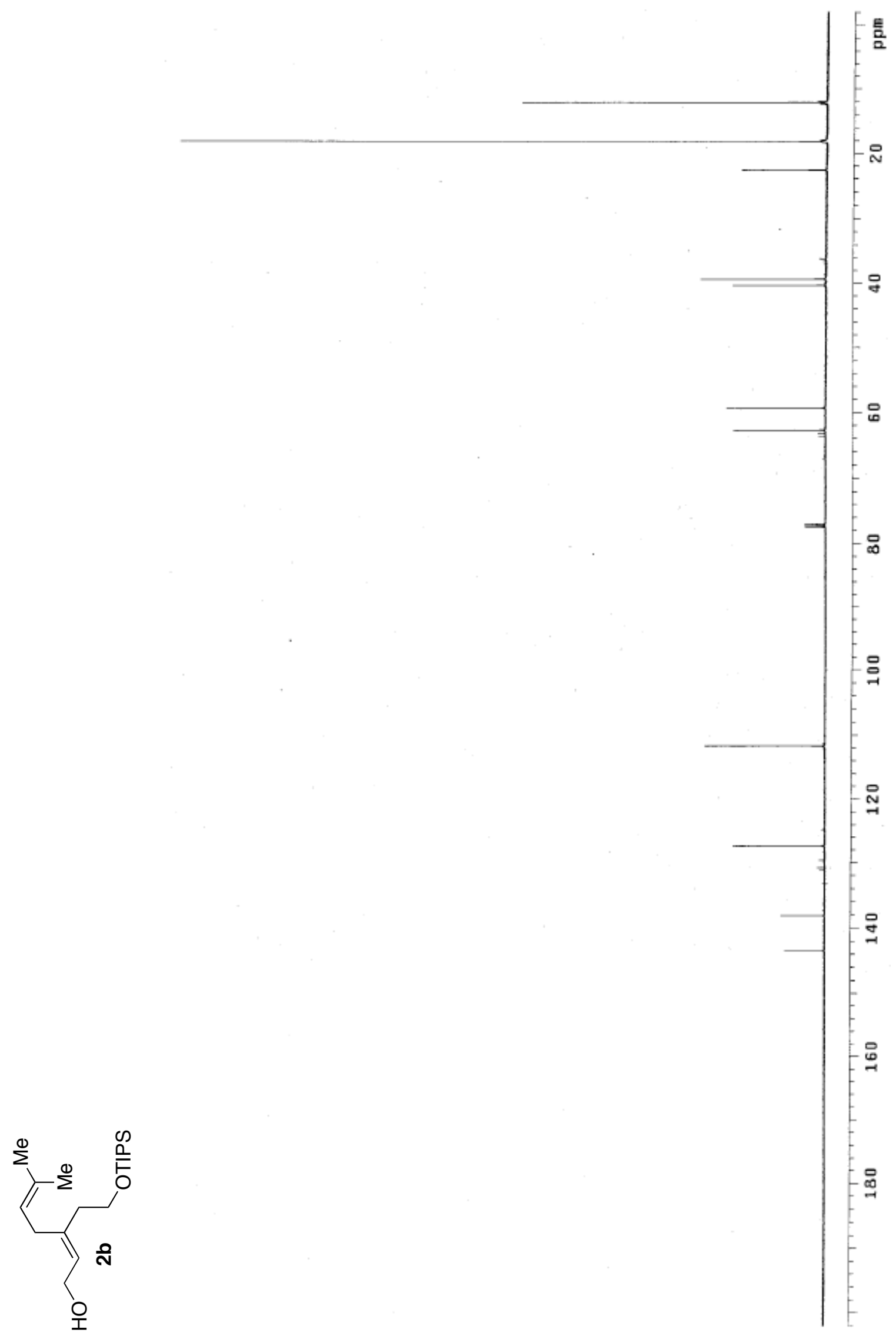




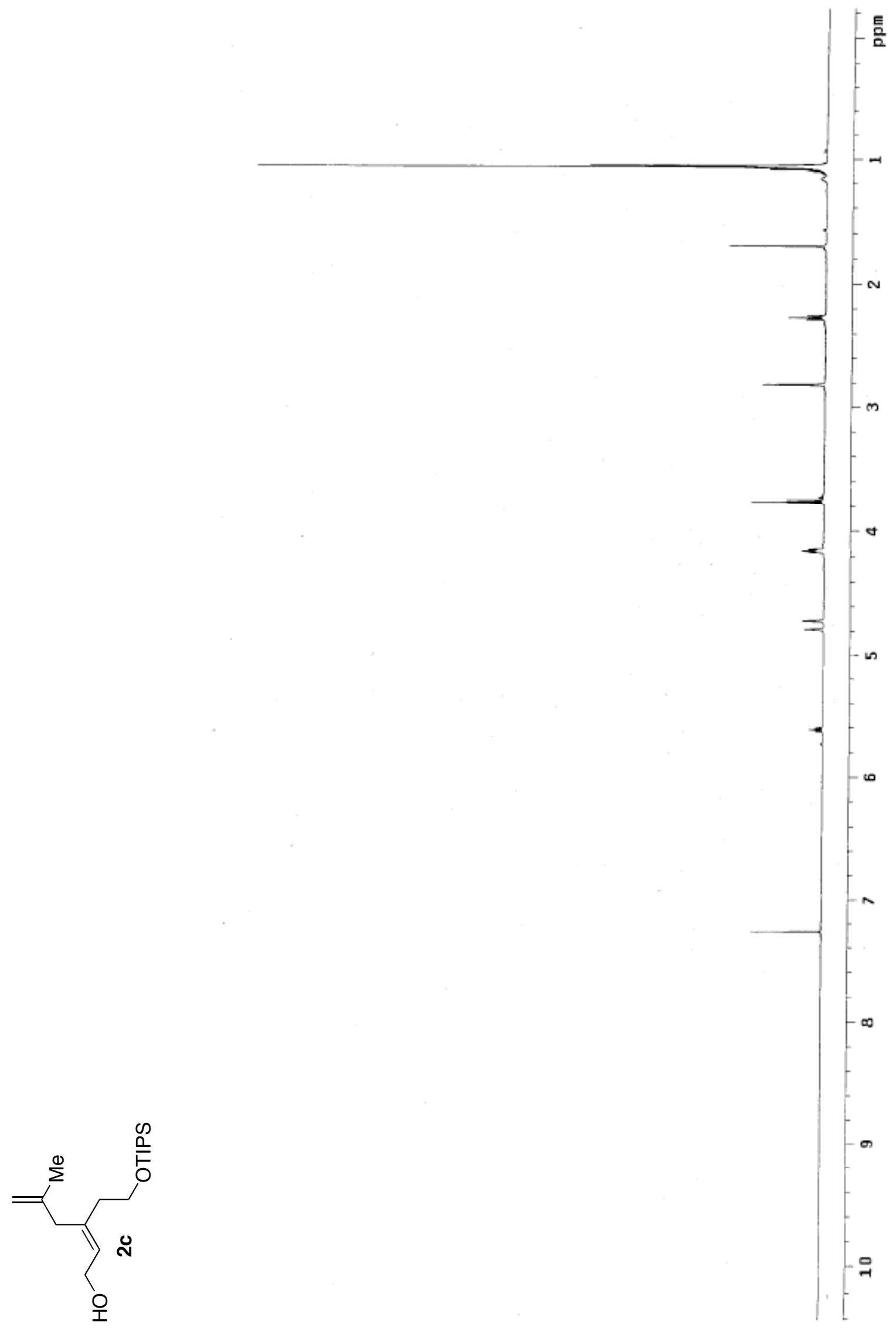




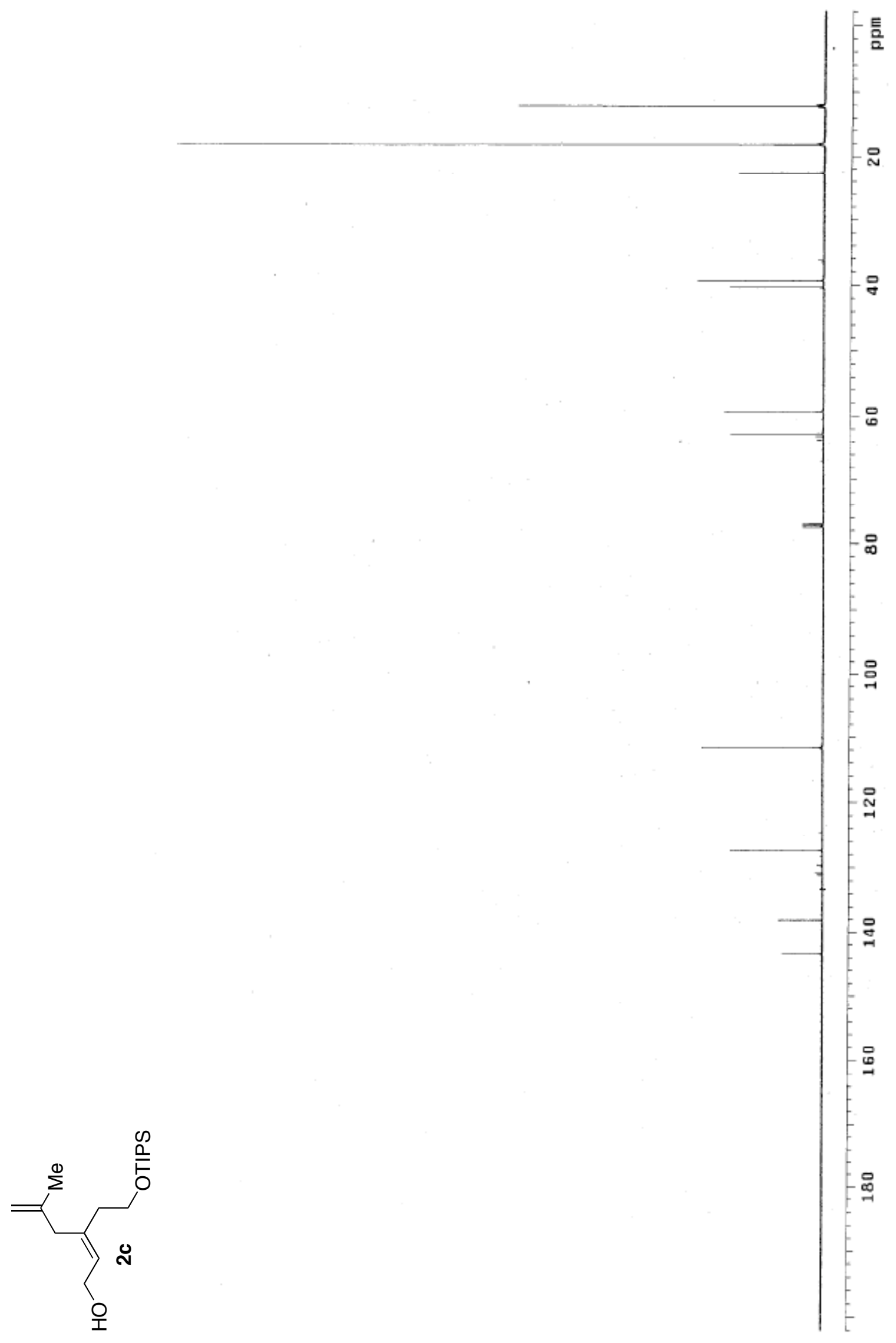

S-30 


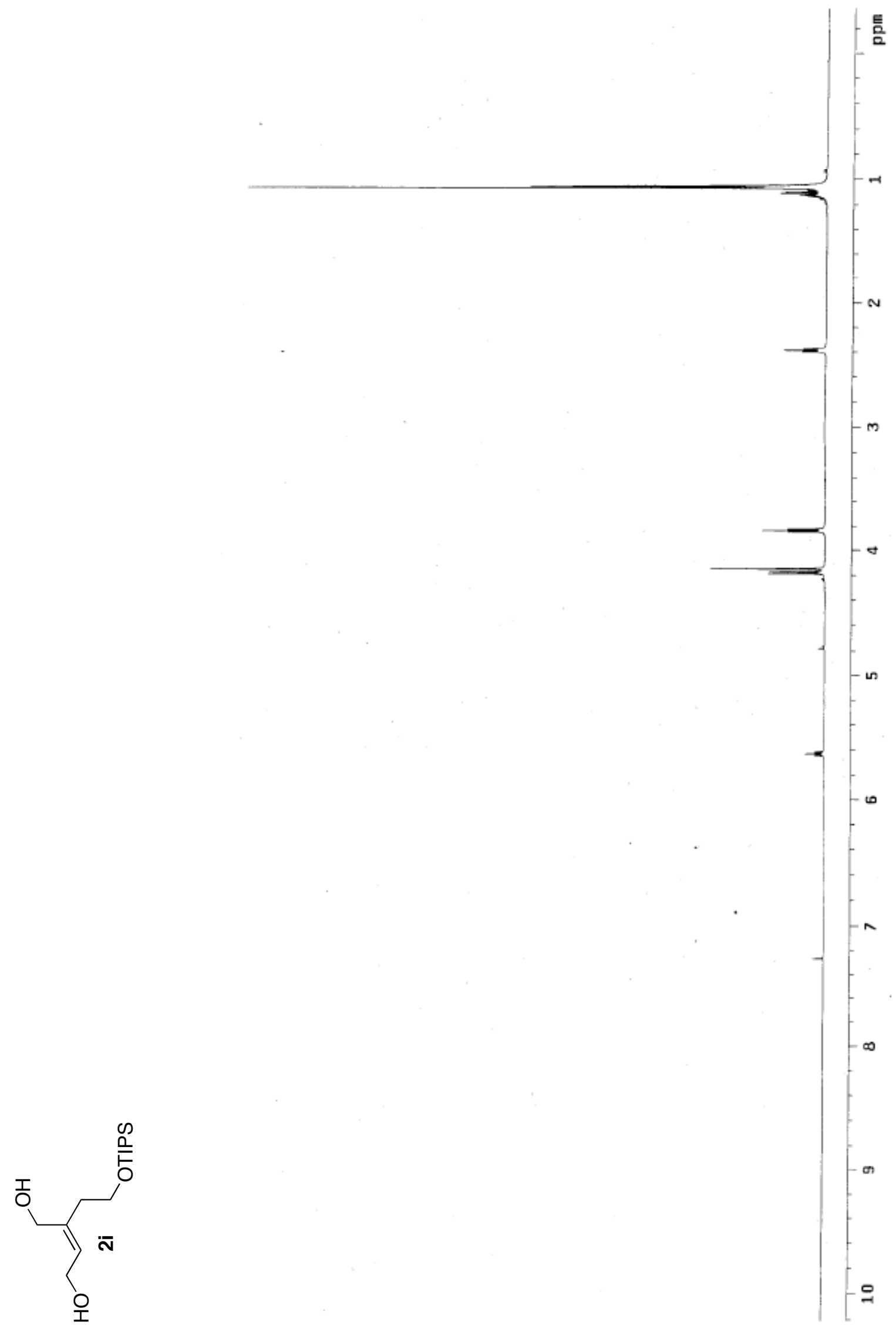

S-31 


$$
\mid
$$




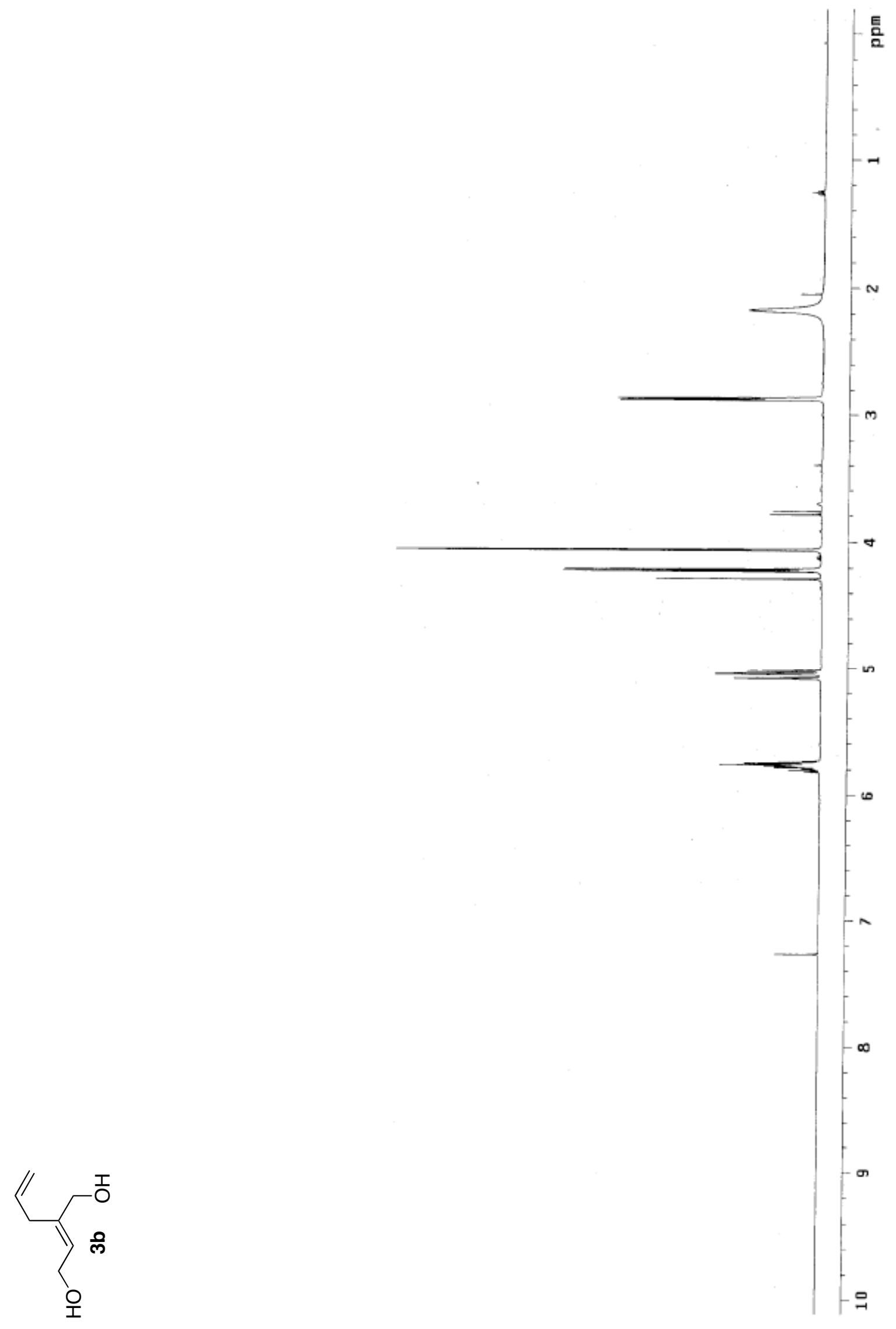

S-33 


$$
1
$$




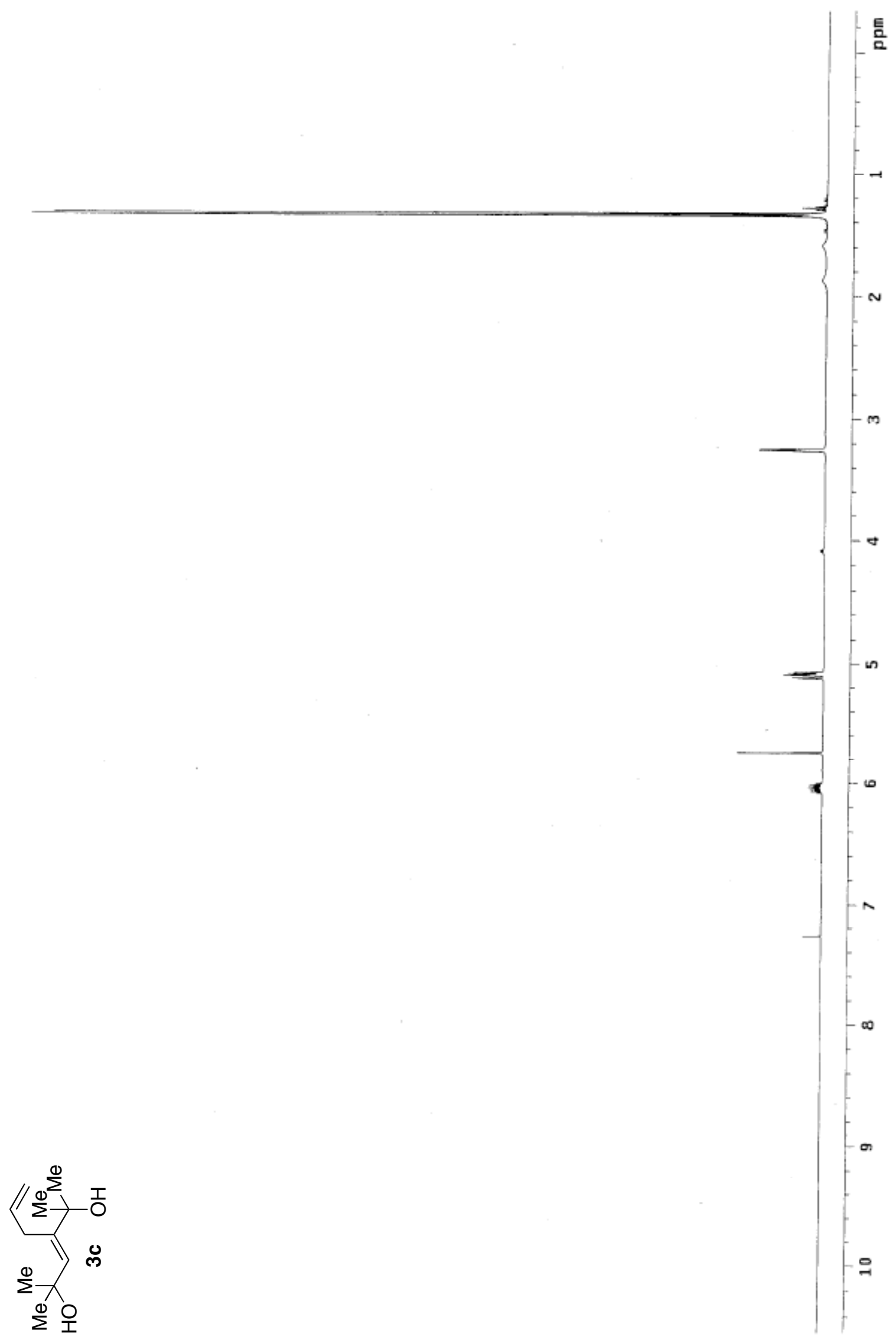

S-35 


$$
1
$$




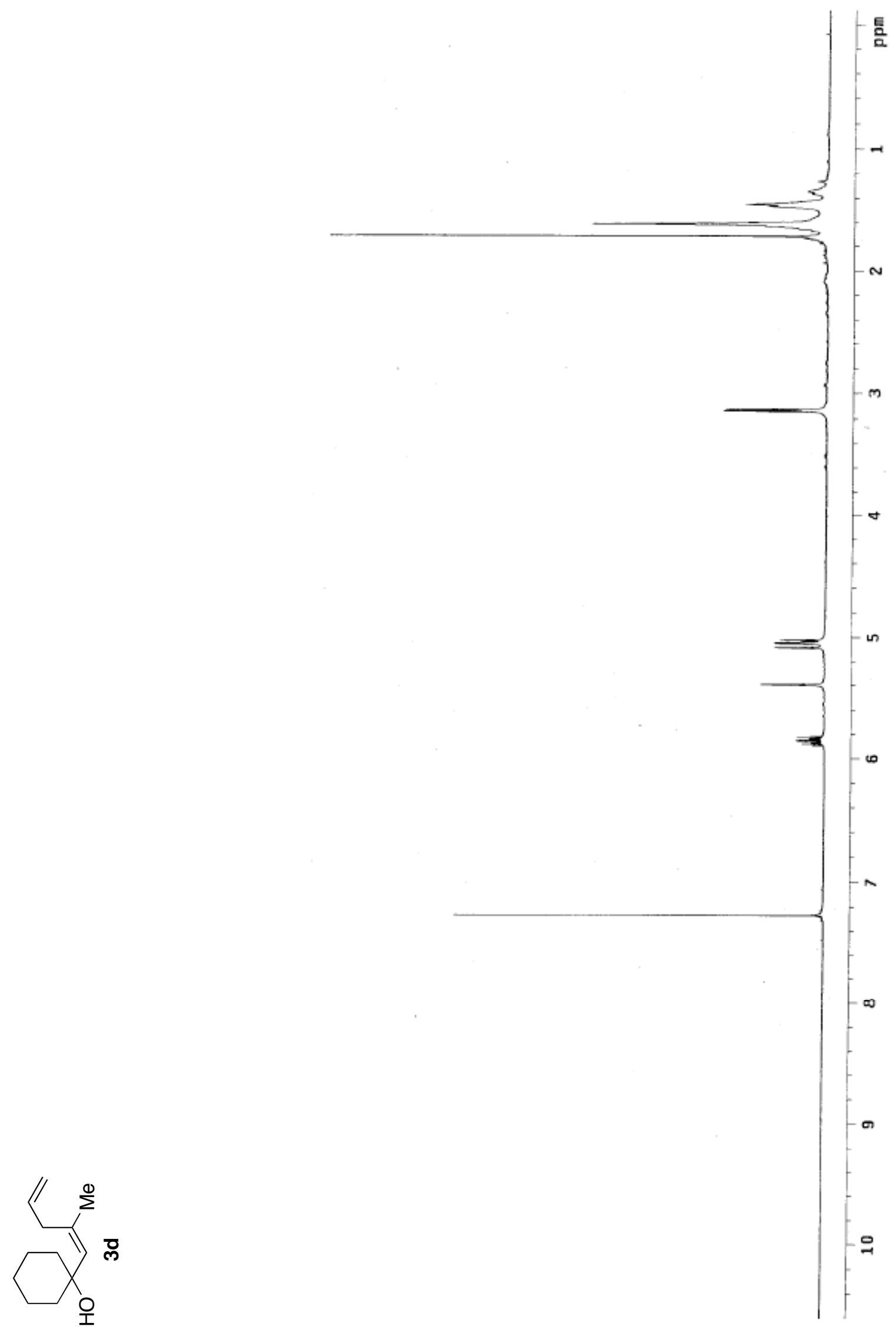

S-37 


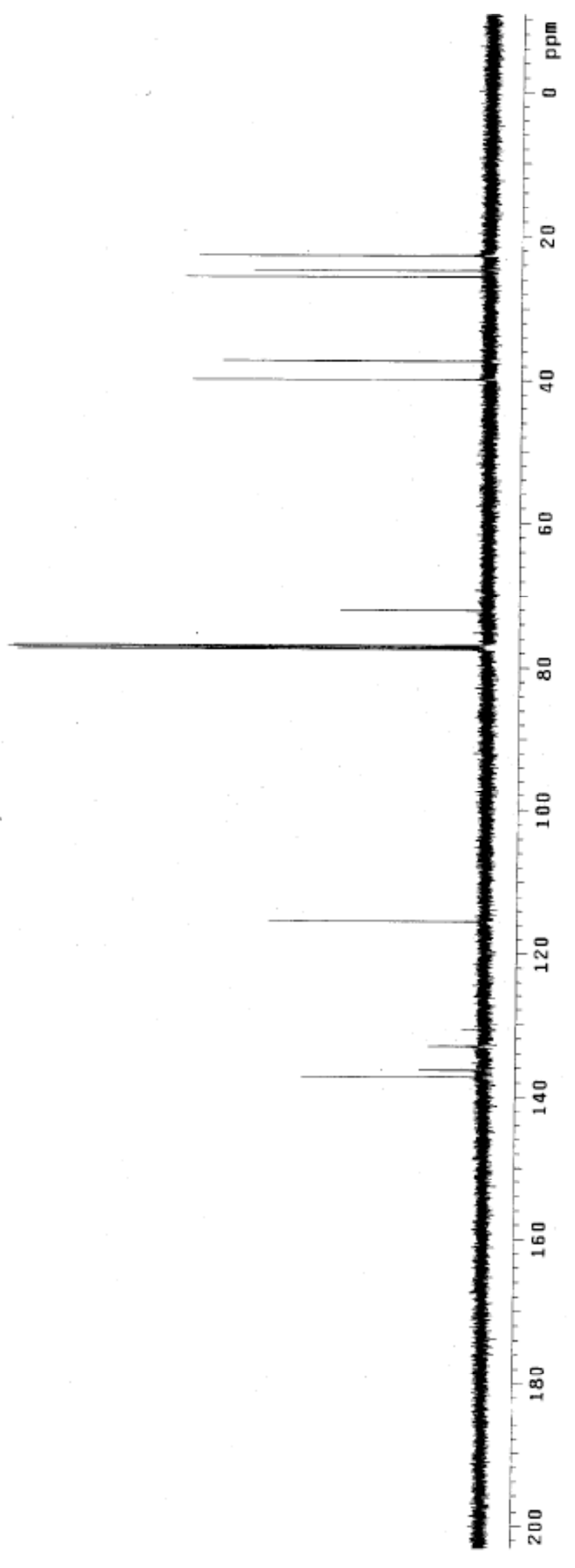

S-38 


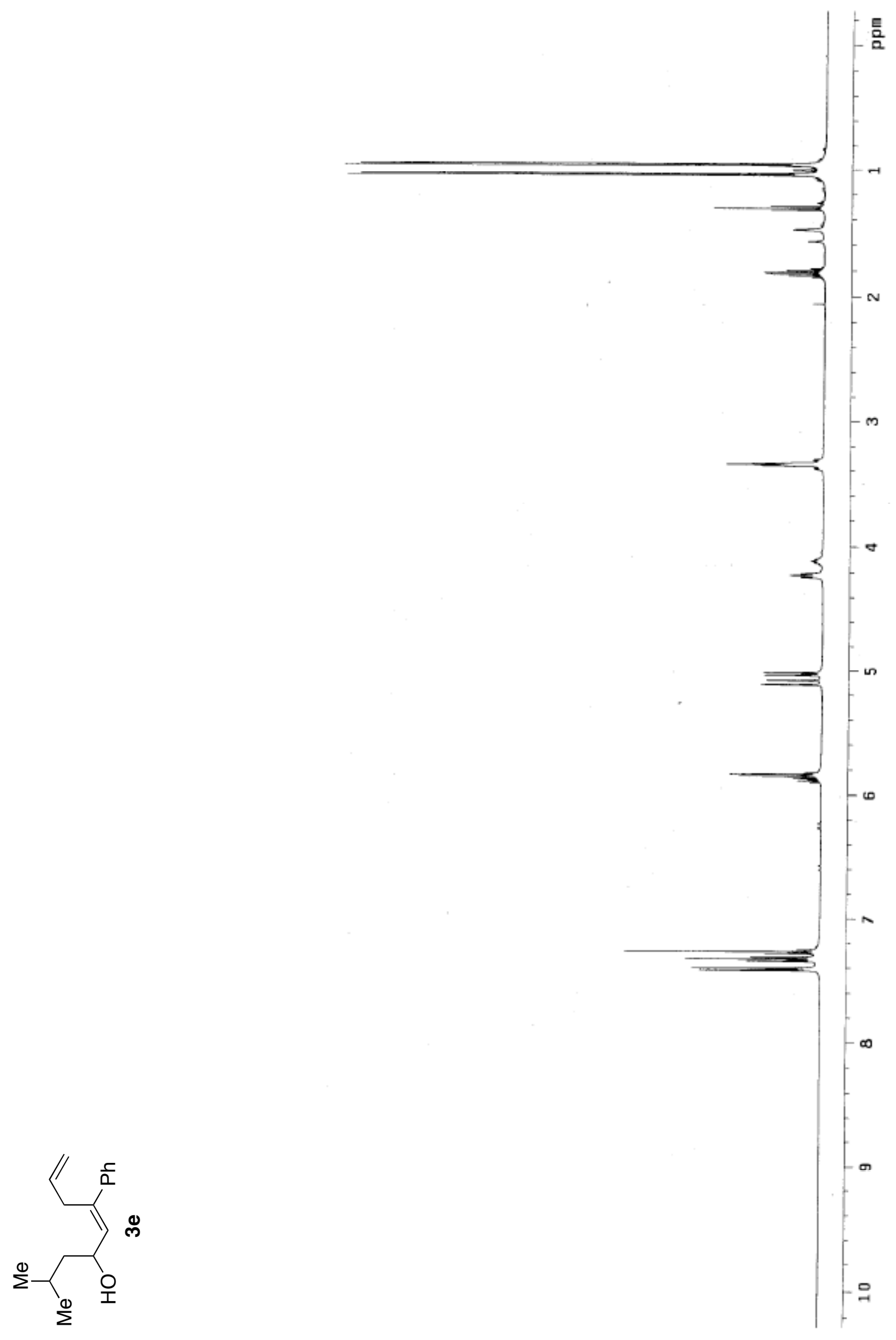

S-39 


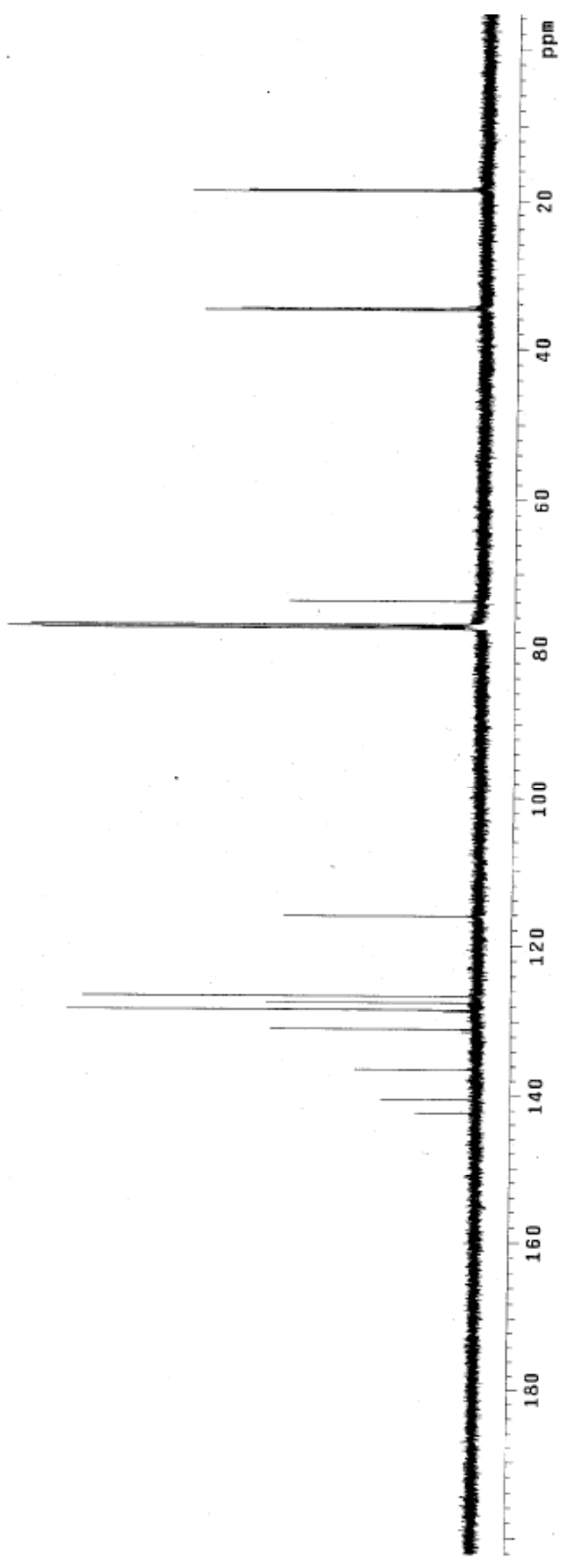

S-40 


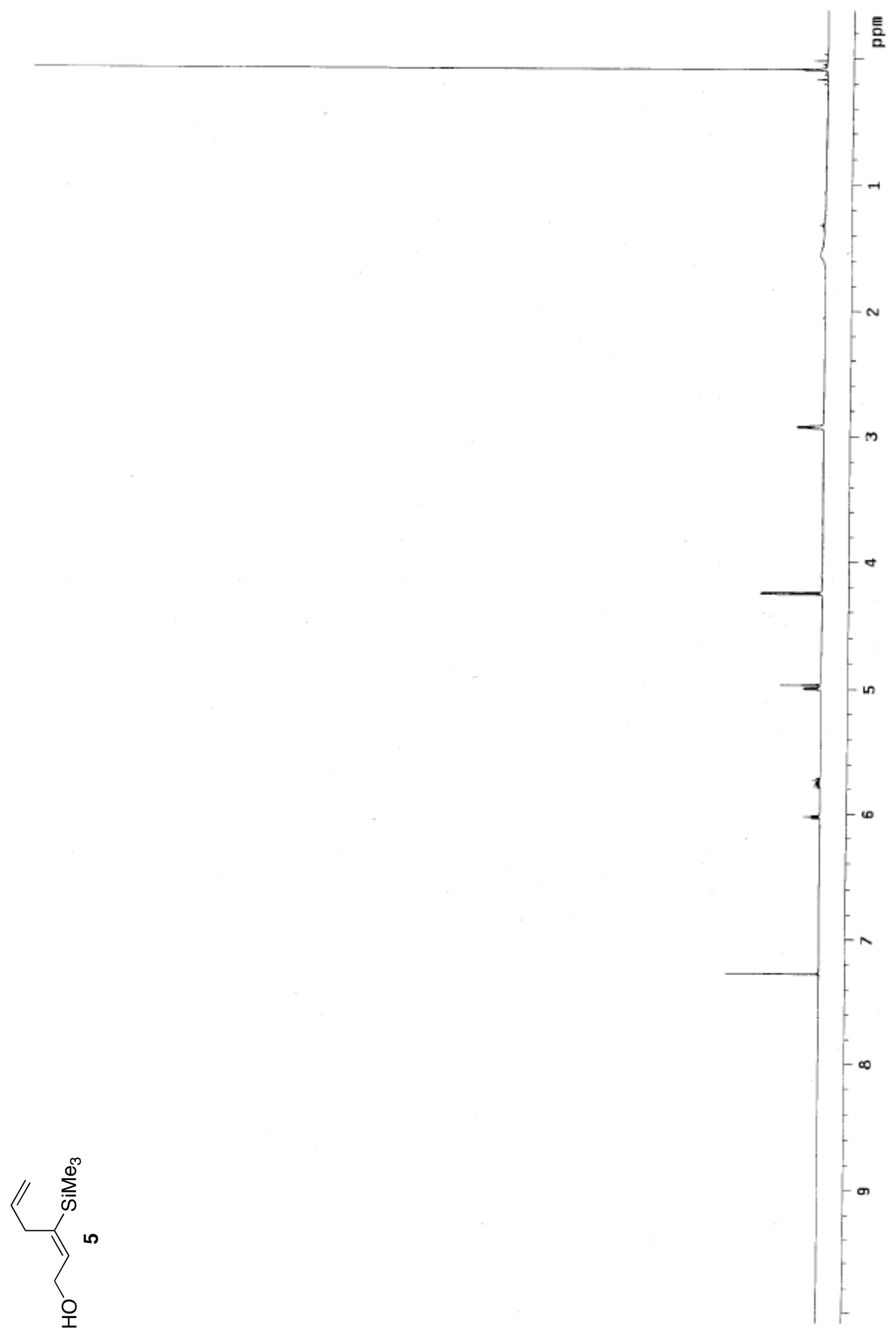




$$
1
$$




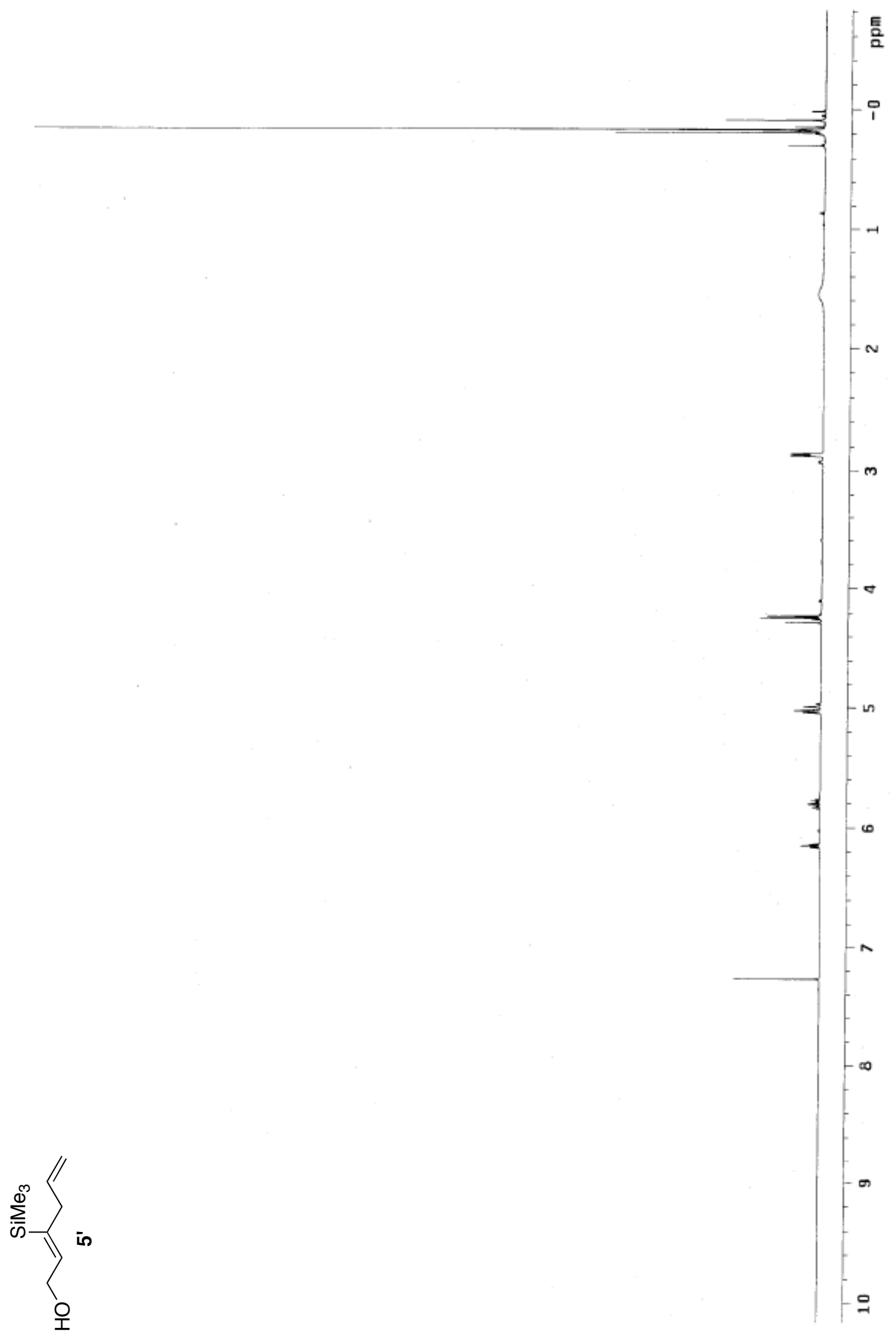




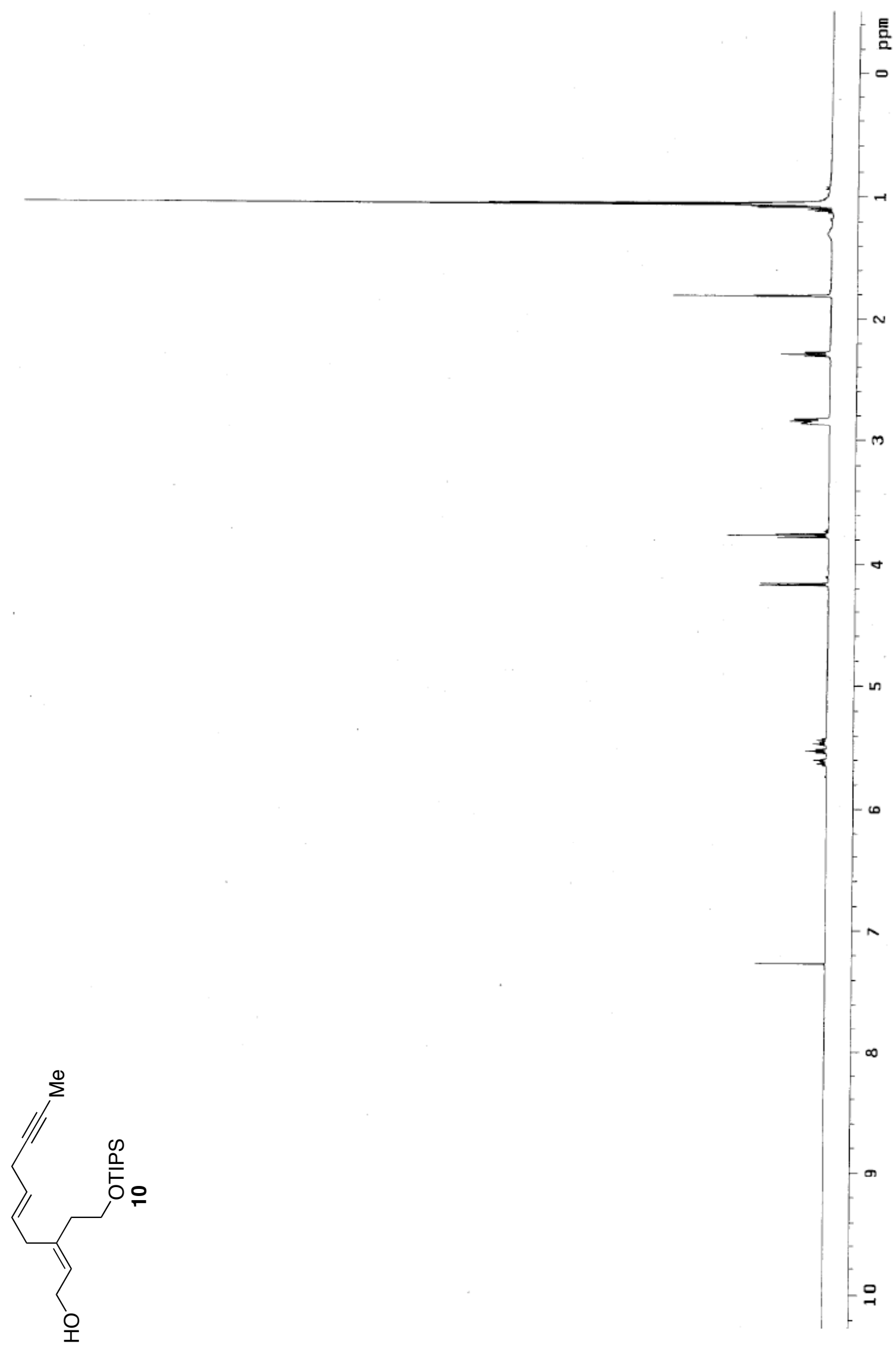




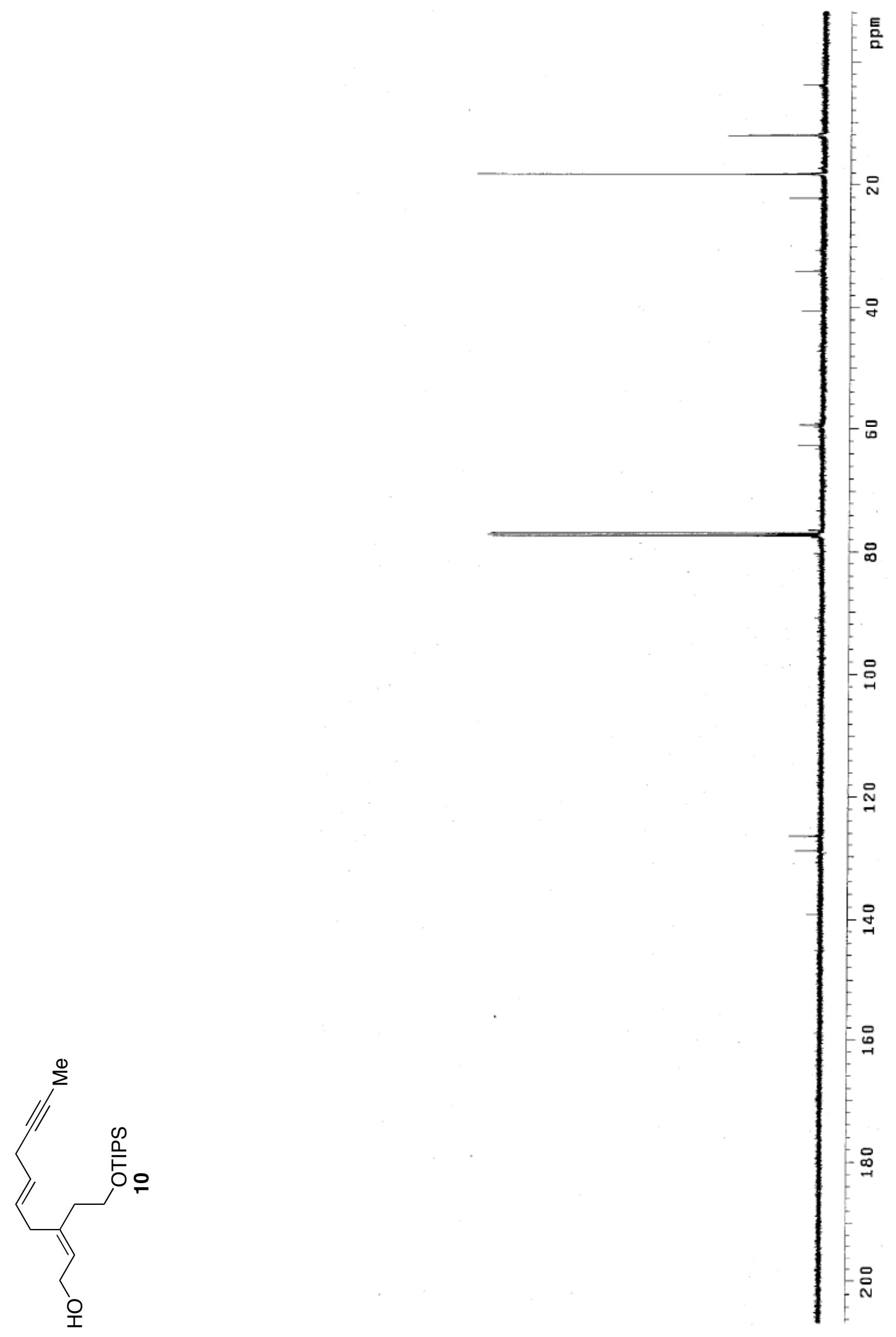




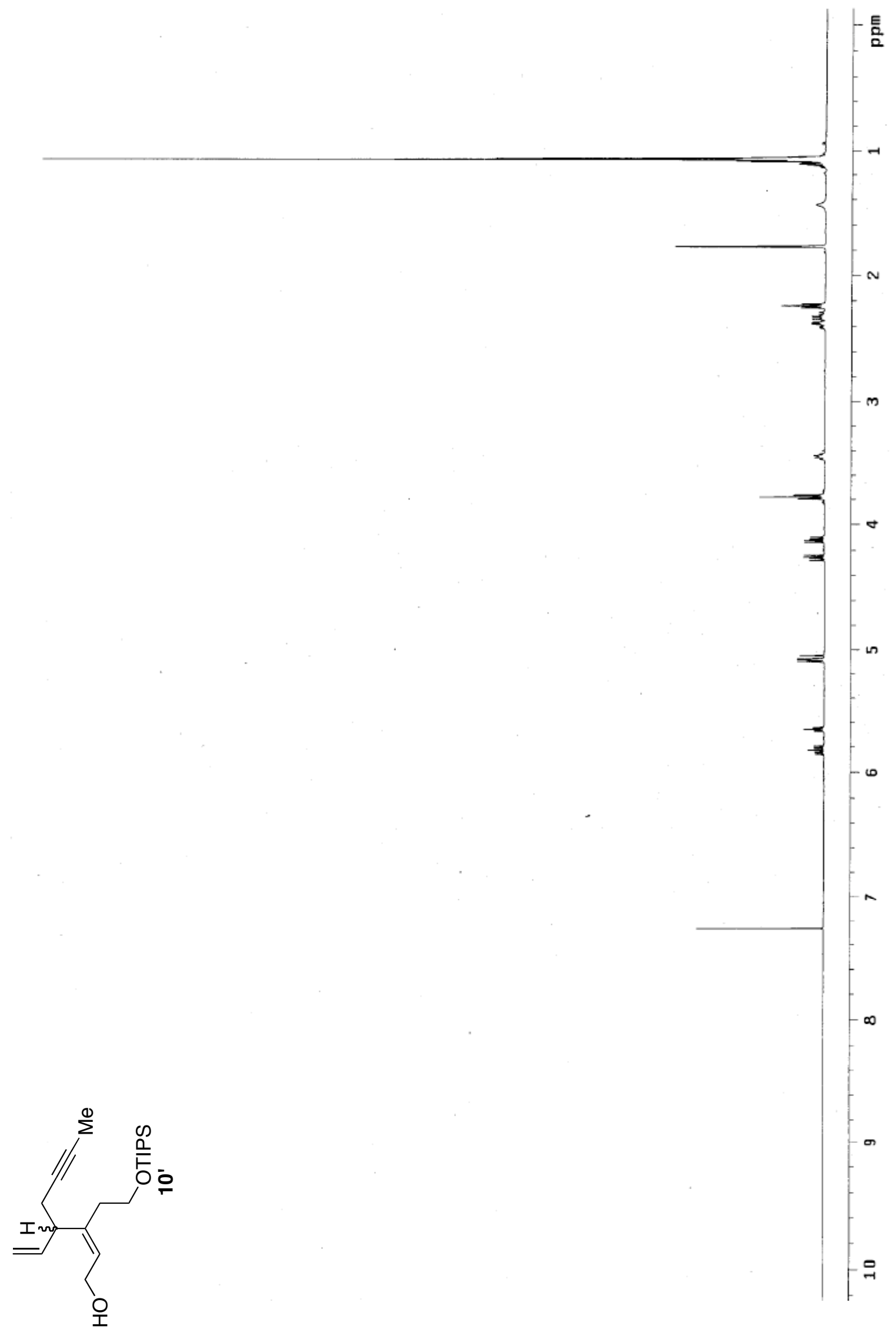




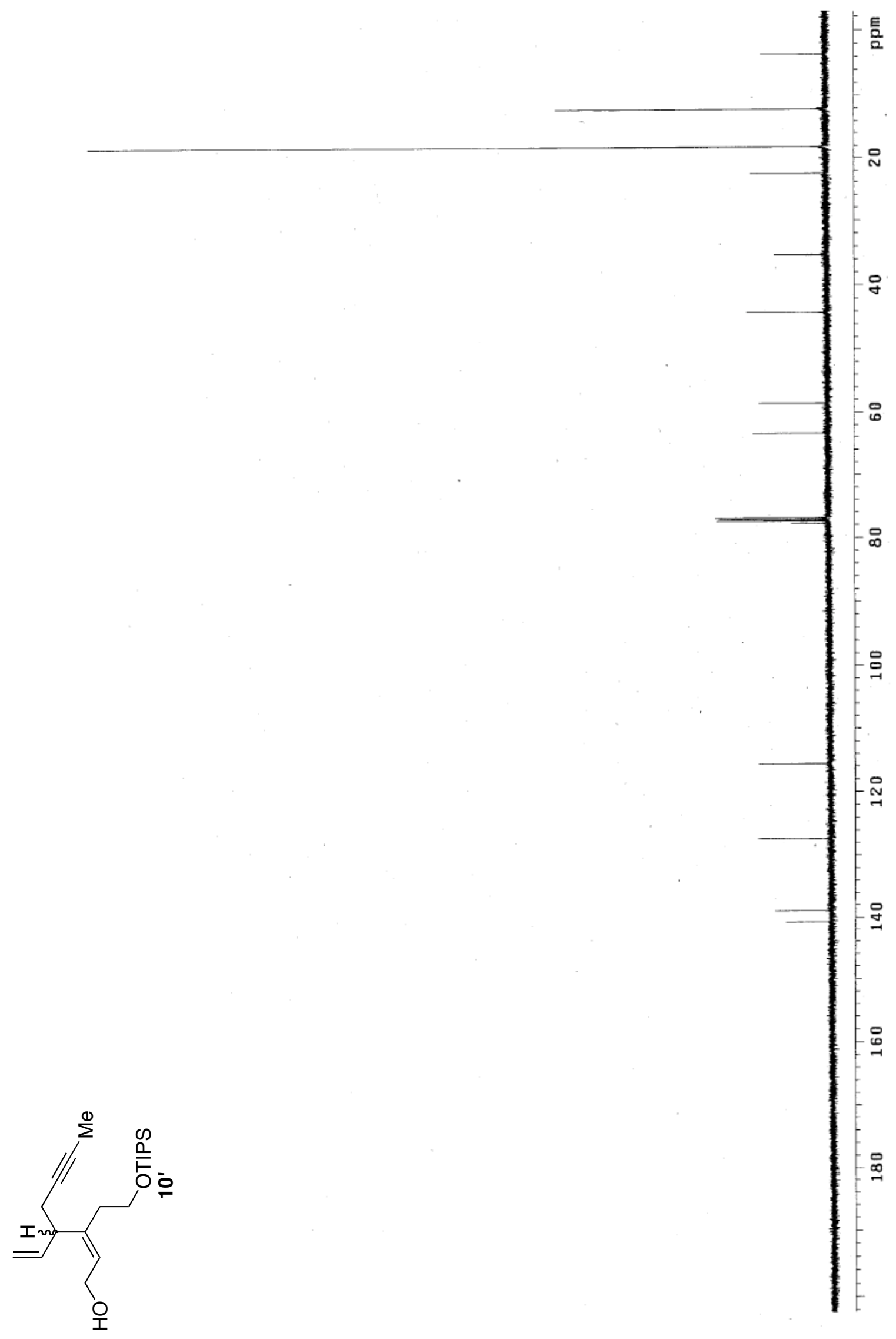




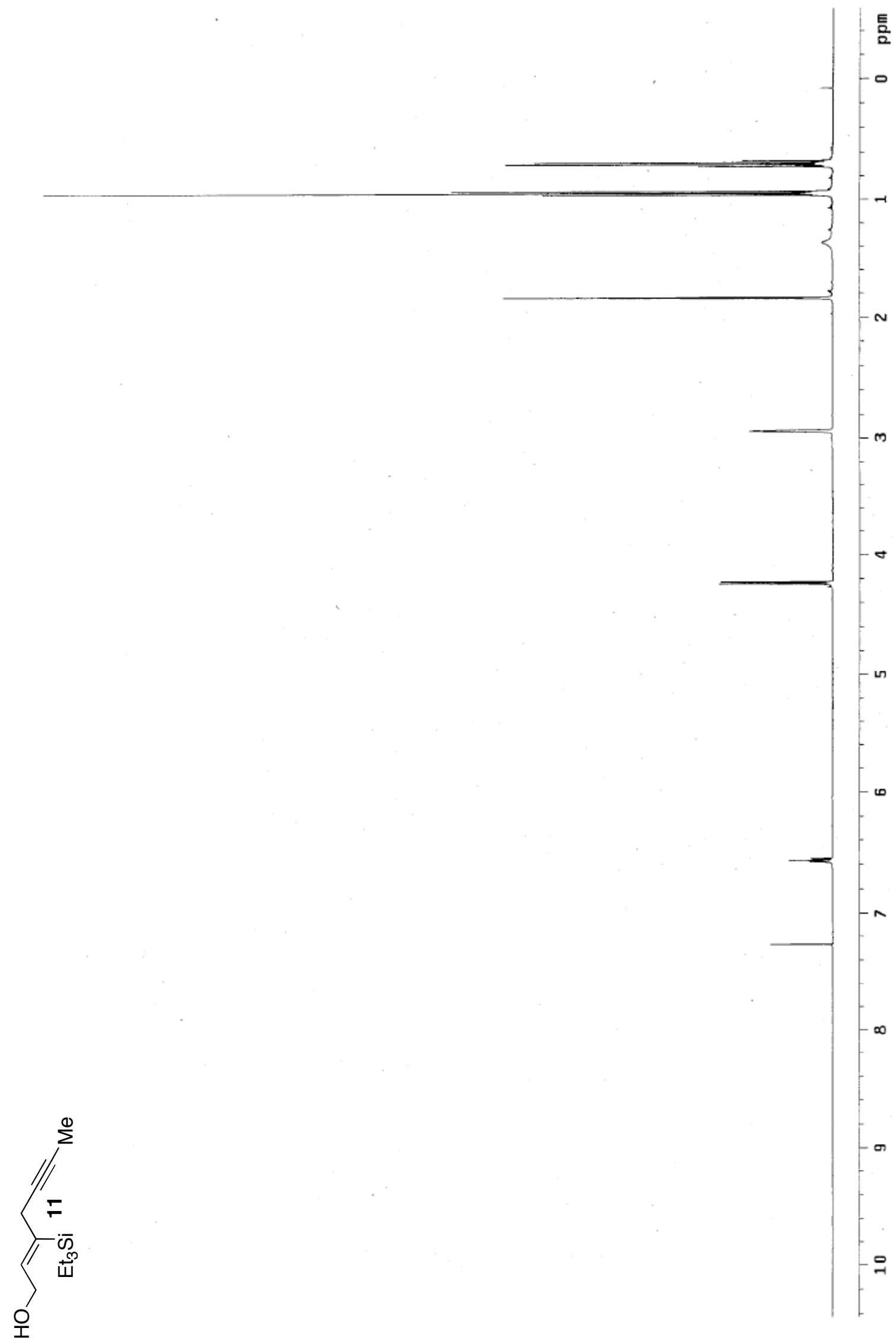




$$
1
$$




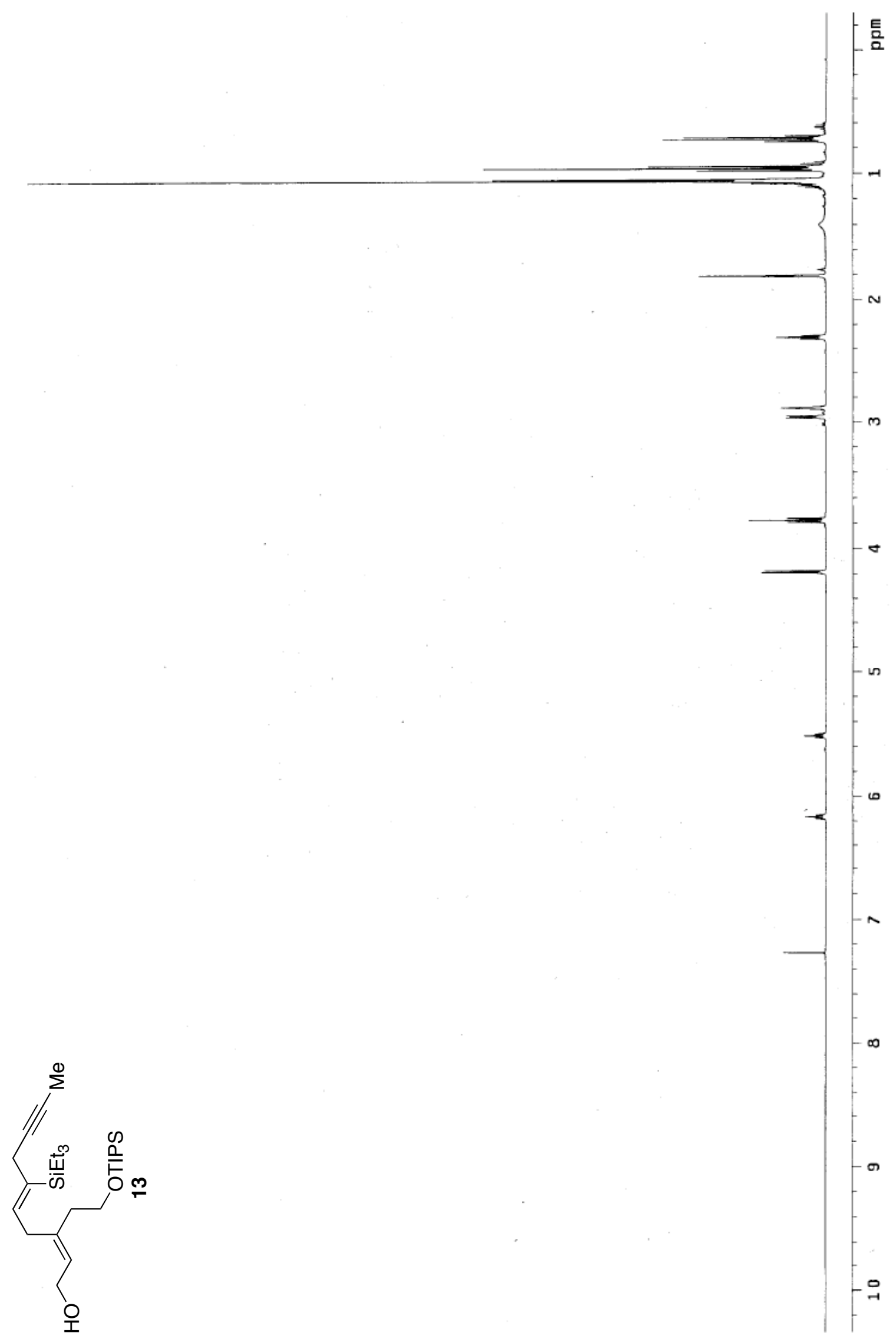




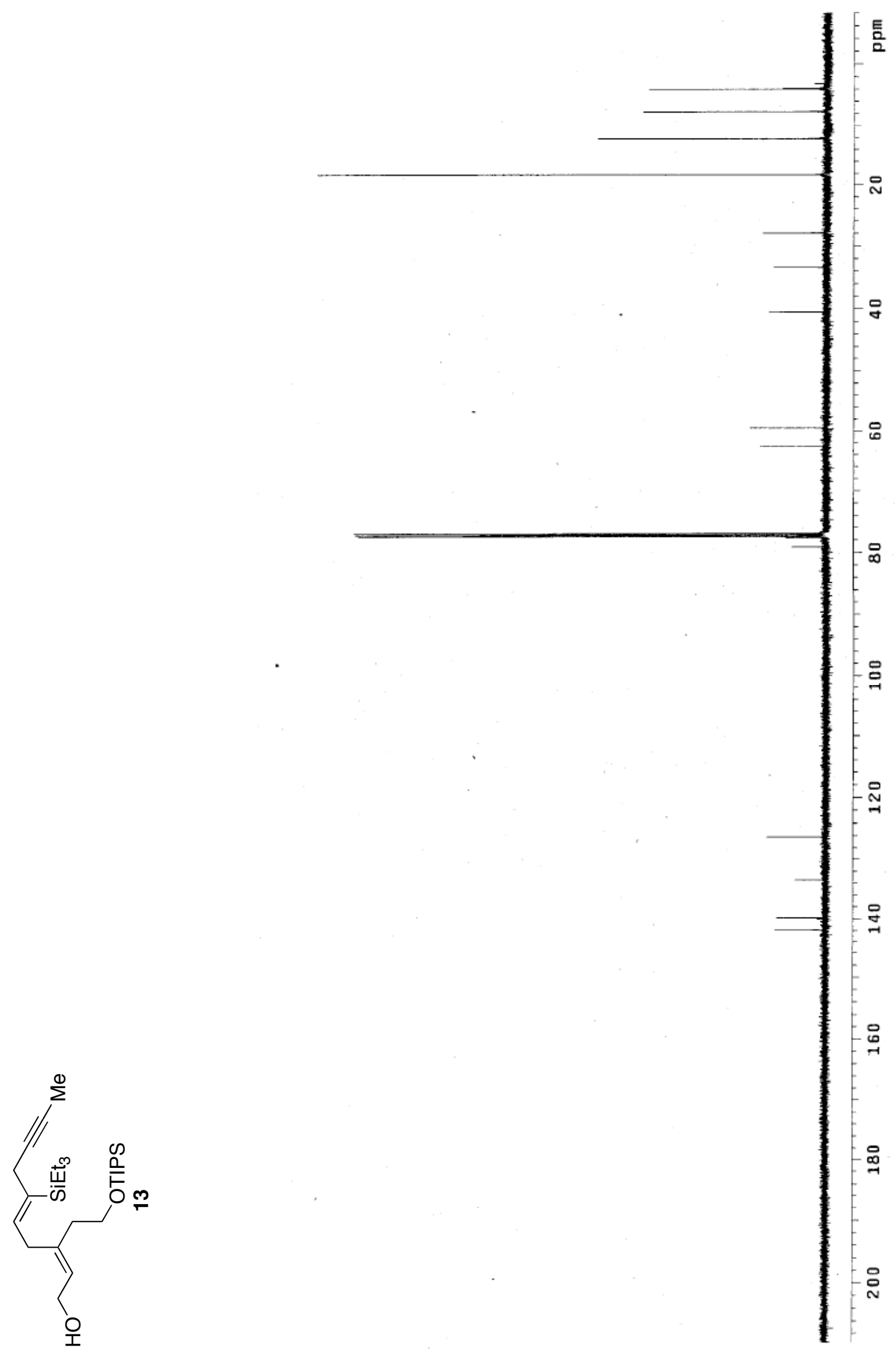

S-51 